TRANSACTIONS OF THE

AMERICAN MATHEMATICAL SOCIETY

Volume 355, Number 4, Pages 1621-1646

S 0002-9947(02)03195-1

Article electronically published on December 4, 2002

\title{
LOGMODULARITY AND ISOMETRIES OF OPERATOR ALGEBRAS
}

\author{
DAVID P. BLECHER AND LOUIS E. LABUSCHAGNE
}

\begin{abstract}
We generalize some facts about function algebras to operator algebras, using the "noncommutative Shilov boundary" or " $C^{*}$-envelope" first considered by Arveson. In the first part we study and characterize complete isometries between operator algebras. In the second part we introduce and study a notion of logmodularity for operator algebras. We also give a result on conditional expectations. Many miscellaneous applications are provided.
\end{abstract}

\section{INTRODUCTION}

The main topic of our paper is the study of linear maps $T: A \rightarrow B$ between operator algebras. By an operator algebra we mean a uniformly closed algebra of operators on a Hilbert space $H$, and we usually assume that such algebras are unital (that is, contain an identity of norm 1), or are approximately unital (that is, contain a contractive two-sided approximate identity (c.a.i.)). Operator algebras may be viewed as noncommutative function algebras. Indeed, every function algebra or uniform algebra on a compact space $K$ containing constant functions is a closed unital subalgebra of the commutative $C^{*}$-algebra $C(K)$, and hence is a unital operator algebra.

It is currently commonly recognized that to study a general subalgebra $A \subset$ $B(H)$ it is necessary not only to take into account the norm, but also the natural norm on the matrix spaces $M_{n}(A) \subset M_{n}(B(H)) \cong B\left(H^{(n)}\right)$. This has been one of the key perspectives of operator space theory, since Arveson's pioneering work; and the present paper may in some sense be regarded as an extended series of observations proceeding from Arveson's papers [6], [7, 5].

Hence we are not interested here in bounded linear transformations; rather we look for the completely bounded maps - where the adjective "completely" means that we are applying our maps to matrices too. Thus if $T: X \rightarrow Y$, then $T$ is completely contractive if and only if

$$
\left\|\left[T\left(x_{i j}\right)\right]\right\| \leq\left\|\left[x_{i j}\right]\right\|
$$

for all $n \in \mathbb{N}$ and $\left[x_{i j}\right] \in M_{n}(X)$. We say that $T$ is completely isometric if $\left\|\left[T\left(x_{i j}\right)\right]\right\|=\left\|\left[x_{i j}\right]\right\|$ for all $n \in \mathbb{N}$ and $\left[x_{i j}\right] \in M_{n}(X)$. In much of this paper

Received by the editors May 15, 2002 and, in revised form, September 4, 2002.

2000 Mathematics Subject Classification. Primary 46L07, 46J10, 46L52, 47L30; Secondary 46E25, 47B33.

This research was supported in part by grants from the National Science Foundation and the University of South Africa. 
we are interested in complete isometries between operator algebras, and in generalizing facts for isometries between function algebras (note that isometries between subspaces of function algebras are the same thing as complete isometries, as may be seen from [40], Theorem 3.8). We now briefly review some of these facts. The classical Banach-Stone theorem characterizes linear isometries between $C(K)$ spaces, that is, between commutative unital $C^{*}$-algebras. There is an analogous result for function algebras (see e.g. p. 144 in [25; 37]). These results have been extended in many directions (see [26] for a survey). The not necessarily surjective isometries between $C(K)$ spaces were characterized by Holsztynski, whereas in the case of function algebras there is the following more general result from 36] (for closely related results see [38, and particularly [2], Section 6):

Theorem 1.1 (36]). A linear map $T: A \rightarrow B$ between uniform algebras is an isometry if and only if $T$ is contractive and there exist a closed subset $E$ of $\partial B$ and two continuous functions $\gamma: E \rightarrow \mathbb{T}$ and $\varphi: E \rightarrow \partial A$, with $\varphi$ surjective, such that for all $y \in E$,

$$
T(f)(y)=\gamma(y) f(\varphi(y))
$$

Here $\mathbb{T}$ is the unit circle, and $\partial B$ is the Shilov boundary of $B$ (which equals $K$ if $B=C(K))$. Informally, the result says that for any isometry $T$ there is a certain 'part $E$ of the space $B$ acts on', such that $T$ restricted to this part has a particularly nice form, a form which amongst other things ensures that $T$ is an isometry. The action of $T$ on the 'complementary part' plays little role in the fact that $T$ is an isometry. If $T$ is unital (that is, $T(1)=1$ ), then the $\gamma$ may be omitted in the theorem, and then $T$ compressed to $E$ is the composition operator $f \mapsto f \circ \varphi$, which is an isometric homomorphism.

For surjective isometries between $C^{*}$-algebras the first 'noncommutative BanachStone theorem' is due to R. V. Kadison [27]. Related isometric results were obtained by Harris, Arazy and Solel and many others (see [3], [39], 1], for example). In Part I of our paper we consider unital or approximately unital operator algebras $A$ and $B$, and (not necessarily surjective) linear maps $T: A \rightarrow B$, and we will establish several criteria which are each equivalent to $T$ being a linear complete isometry. These are generalizations of 1.1 because, as we said earlier, any function algebra is a unital operator algebra; and moreover, isometries between function algebras are completely isometric. In the result above we saw the importance of the Shilov boundary; in the noncommutative case we will need to use the noncommutative Shilov boundary, or more specifically the $C^{*}$-envelope $C_{e}^{*}(A)$, of an operator algebra $A$. This will be described in more detail towards the end of this introduction.

One such characterization proceeds as follows. Suppose that $A, B$ are operator algebras, and suppose that $B$ is a subalgebra of a $C^{*}$-algebra $C$. Suppose that $T: A \rightarrow B$ is a linear map. For the purposes of additional clarity, here we assume that $A, B$ and $T$ are unital (in the general case one needs to add a partial isometry and a second projection $q$ to the statement that follows). Then $T$ is a complete isometry if and only if there is a projection $p$ in $B\left(H_{u}\right)$, where $H_{u}$ is the Hilbert space of the universal representation of $C$, such that, firstly,

$$
p T(\cdot)=T(\cdot) p=p T(\cdot) p=\pi(\cdot)
$$

for a completely isometric homomorphism $\pi$ that is the restriction to $A$ of a 1-1 *-representation $C_{e}^{*}(A) \rightarrow B\left(p H_{u}\right)$; and secondly, the map $S=(1-p) T(\cdot)(1-p)$ 
is a complete contraction. This is saying that, up to a choice of orthonormal bases, $T$ is of 'block diagonal' form

$$
\left[\begin{array}{cc}
\pi(\cdot) & 0 \\
0 & S(\cdot)
\end{array}\right]
$$

In Part I we also consider particularly interesting subclasses of complete isometries between operator algebras. In particular, we investigate a class which was introduced in the commutative case by Matheson under the name type 1 isometry.

As we have said, an important tool is the $C^{*}$-envelope $C_{e}^{*}(A)$ of an operator algebra $A$. However, there are two basic obstacles associated with this tool, which we address (with very partial success) in the second half of our paper. The first obstacle is that of identifying $C_{e}^{*}(A)$ for a given operator algebra $A$. Following the classical case, we introduce a notion of logmodularity for operator algebras, and show that for a $\operatorname{logmodular}$ subalgebra $A$ of a $C^{*}$-algebra $B$, the $C^{*}$-envelope of $A$ is $B$. We develop some basic theory of logmodular algebras, giving for example a result which is a noncommutative version of the uniqueness of representing measures. Indeed, this result ensures that a completely contractive homomorphism $\theta$ defined on a logmodular operator algebra has at most one completely contractive extension to $C_{e}^{*}(A)$. This extension will be completely isometric if $\theta$ is.

A good example of a logmodular algebra in the commutative case is the Hardy space $H^{\infty}(\mathbb{D})$; and in the noncommutative case the noncommutative $H^{\infty}$ algebras introduced by Arveson (also known as finite maximal subdiagonal algebras). In section 5 we make a few observations about these algebras using the facts in the previous paragraph. In this section we also prove some general results on conditional expectations of non-selfadjoint operator algebras.

The second obstacle associated with the $C^{*}$-envelope arises when $C_{e}^{*}(A)$ is rather complicated. Again a good example of this is $H^{\infty}(\mathbb{D})$. In light of the known characterizations of isometries of other common function algebras in terms of composition operators (see [48], for example), one would hope for a characterization in which analytic self-maps of the open disk play a major role (as opposed to self-maps of the largely unhelpful Shilov boundary). However, for this purpose our results on complete isometries from Part I are often not helpful. To illustrate this point we recall that there is no such known characterization of isometries $H^{\infty}(\mathbb{D}) \rightarrow H^{\infty}(\mathbb{D})$. In section 3 we make a few observations concerning isometries of $H^{\infty}(\mathbb{D})$. Our main interest, however, is in the noncommutative versions, and we are currently working with A. Matheson in this direction.

We end this introduction with some notation and basic facts. For more details on operator spaces we refer the reader to [19], [40], [42]. We write $H, K, L$ for Hilbert spaces. Perhaps confusingly, we will use the symbols $I, J, K$ for ideals in a $C^{*}$-algebra. All ideals are taken to be uniformly closed. A projection on a Hilbert space, or in a $C^{*}$-algebra, will mean an orthogonal projection. Otherwise we use the word projection for a linear self-map $P$ with $P \circ P=P$. If $Y$ is a subspace of $X$, we write $q_{Y}$ or $q$ for the canonical quotient map $X \rightarrow X / Y$. For a set of operators on a Hilbert space, or for a subset of an operator algebra, we write $S^{*}$ for the subset consisting of adjoints of elements of $S ; S_{+}$for the set of $x \in S$ with $x \geq 0$; and $S^{-1}$ for the invertible elements of $S$ whose inverse is also in $S$. We write $|b|$ for $\left(b^{*} b\right)^{\frac{1}{2}}$. To avoid possible confusion, the Banach dual of a Banach space $X$ will be denoted by $X^{\star}$. The canonical map $X \rightarrow X^{\star \star}$ is written as ^^. 
If $X, Y$ are subspaces of a Banach algebra, we write $X Y$ for the uniform closure of the set of finite sums of products of the form $x y$ for $x \in X, y \in Y$.

An operator system is a unital selfadjoint subspace $X$ of a unital $C^{*}$-algebra or of $B(H)$ (i.e., $X$ contains the identity element, and $x \in X$ if and only if $x^{*} \in X$ ). A unital operator space has the same definition, except we do not require that $X^{*}=$ $X$. The appropriate morphisms between operator systems are unital completely positive maps. Rather than state the (obvious) definition of a completely positive map, we will simply use the fact that a unital linear map $S$ between operator systems is completely contractive if and only if it is completely positive, in which case it is *-linear, i.e., $S\left(v^{*}\right)=S(v)^{*}$. See [6], 40] or [17] for proofs.

Lemma 1.2 (Arveson, see e.g. 17]). Suppose that $V$ is a subspace of an operator system that contains the identity element, and suppose that $\psi: V \rightarrow B(K)$ is a unital contraction with range $W$. Then there exists a unique positive map between the operator systems $V+V^{*}$ and $W+W^{*}$ that extends $\psi$. If $\psi$ is a unital complete contraction (resp. unital complete isometry), then the unique positive extension to $V+V^{*}$ is a completely positive map (resp. a unique complete order isomorphism) between the operator systems $V+V^{*}$ and $W+W^{*}$.

As an aside, we note that in the context of $H^{p}$ spaces, the last result seems to translate to a principle whereby composition operators on $H^{p}(\mathbb{D})$ spaces may be extended to positive integral operators on the enveloping $L^{p}(\mathbb{T})$-spaces. (See [47.)

Now if $T: X \rightarrow A$ and $S: X \rightarrow B$ are unital complete isometries of an abstract unital operator space $X$ into $C^{*}$-algebras $A$ and $B$, then by the above result there exists a unique complete order isomorphism between the operator systems $T(X)+T(X)^{*}$ and $S(X)+S(X)^{*}$ which extends the map $S \circ T^{-1}$ from $T(X) \rightarrow$ $S(X)$. As a corollary of this, one may easily check that if $A$ is an abstract unital operator algebra, then the two spaces $\Delta(A)=A \cap A^{*}$ (the diagonal of $A$ ) and $A+A^{*}$ are 'well defined' independently of the particular $C^{*}$-algebra containing $A$ as a unital subalgebra. Note that $\Delta(A)$ is a $C^{*}$-algebra (indeed, it is a $W^{*}$-algebra if $A$ is a 'weak* closed operator algebra'), and $A+A^{*}$ is an operator system. See also [3] for some interesting related facts.

If $X$ is a unital operator space, then there exists a $C^{*}$-envelope of $X$, namely a pair $(B, j)$ consisting of a unital $C^{*}$-algebra $B$ and a unital complete isometry $j: X \rightarrow B$ whose range generates $B$ as a $C^{*}$-algebra, with the following universal property: For any other pair $(A, i)$ consisting of a unital $C^{*}$-algebra and a unital complete isometry $i: X \rightarrow A$ whose range generates $A$ as a $C^{*}$-algebra, there exists a (necessarily unique, unital, and surjective) ${ }^{*}$-homomorphism $\pi: A \rightarrow B$ such that $\pi \circ i=j$. The kernel $\operatorname{Ker} \pi$ is called the Shilov boundary ideal of $X$ in $A$. The existence of the $C^{*}$-envelope with the universal property above we call the Arveson-Hamana theorem [6], 22], and as customary we write $C_{e}^{*}(X)$ for $B$ or $(B, j)$ (it is essentially unique, by the universal property). Note that this use of the term 'Shilov boundary' matches the classical situation, where the Shilov boundary $\partial A$ of a unital subspace $X \subset C(K)$ is the smallest closed subset of $K$ on which each member of $X$ attains its uniform norm. It thus corresponds to the largest ideal of $C(K)$ for which the canonical map $X \rightarrow C(K) / J$ is isometric (and therefore, by an earlier remark, completely isometric).

If $A$ is a unital operator algebra, then it is easy to see that $j$ in the last paragraph is forced to be a homomorphism. Thus $A$ may be considered a unital subalgebra of $C_{e}^{*}(A)$. If $A$ is an approximately unital operator algebra, then one may define 
$C_{e}^{*}(A)$ to be the $C^{*}$-algebra generated by $A$ inside the $C^{*}$-envelope of the unitization of $A$. One easily sees that $C_{e}^{*}(A)$ has the desired universal property.

For the purposes of this paper we define a triple system to be a (uniformly closed) subspace $X$ of a $C^{*}$-algebra such that $X X^{*} X \subset X$. The important structure on a triple system is the triple product $x y^{*} z$. A triple subsystem is a uniformly closed vector subspace of a triple system that is closed under this triple product.

It is well known that $X X^{*} X=X$ for a triple system $X$. Also, it is clear that $X X^{*}$ and $X^{*} X$ are $C^{*}$-algebras, which we will call the left and right $C^{*}$-algebras of $X$ respectively, and $X$ is a $\left(X X^{*}\right)-\left(X^{*} X\right)$-bimodule. A linear map $T: X \rightarrow Y$ between triple systems is a triple morphism if $T\left(x y^{*} z\right)=T(x) T(y)^{*} T(z)$ for all $x, y, z \in X$. Triple systems are operator spaces, and triple morphisms behave very similarly to ${ }^{*}$-homomorphisms between $C^{*}$-algebras: triple morphisms are automatically completely contractive and have closed range. A triple morphism is completely isometric if it is 1-1. The kernel of a triple morphism on $X$ is a triple ideal (that is, a uniformly closed $\left(X X^{*}\right)-\left(X^{*} X\right)$-subbimodule). The quotient of a triple system by a triple ideal is a triple system in an obvious way. If one factors a triple morphism by its kernel, one obtains a 1-1 triple morphism on the quotient triple system. These results are all rather old, may be found in 23, and are related to results of Harris and Kaup.

If $X$ is any operator space, then, there exists a triple envelope of $X$, namely a pair $(Z, j)$ consisting of a triple system $Z$ and a linear complete isometry $j: X \rightarrow Z$ whose range generates $Z$ as a triple system (that is, there exists no nontrivial closed triple subsystem containing $j(X)$ ), with the following universal property: For any other pair $(W, i)$ consisting of a triple system and a complete isometry $i: X \rightarrow W$ whose range generates $W$ as a triple system, there exists a (necessarily unique and necessarily surjective) triple morphism $\pi: W \rightarrow Z$ such that $\pi \circ i=j$. Again one may call $\operatorname{Ker} \pi$, which is a triple ideal in $W$, the Shilov boundary ideal of $X$. This theorem, dating from the $80 \mathrm{~s}$, is due to Hamana [23], and we write $\mathcal{T}(X)$ for $Z$ or $(Z, j)$ (as before, it is essentially unique, by the universal property). The triple envelope or $C^{*}$-envelope is usually defined as a subspace of the injective envelope $(I(X), j)$. We will also need this latter envelope, but since our use of it is quite limited we will not take the time to define it here, and instead refer the reader to [23] or 19] for more details (the forthcoming revision of [40] focuses extensively on this topic too). See also [10] for a more thorough discussion of the concepts in the last several paragraphs.

Part I. Characterizations of complete isometries

\section{General Banach-Stone theorems BETWEEN NON-SELFADJOINT OPERATOR ALGEBRAS}

Suppose that $T: A \rightarrow B$ is a surjective unital complete isometry between unital operator algebras $A$ and $B$. Then, by the universal property of the $C^{*}$-envelope, $T$ extends to a unital complete isometry $\tilde{T}$ between the $C^{*}$-envelopes $C_{e}^{*}(A)$ and $C_{e}^{*}(B)$. By the universal property $\tilde{T}$ is a ${ }^{*}$-homomorphism (or this may be seen by a simpler Banach-Stone theorem for $C^{*}$-algebras such as 5.2.3 in [19]). Consequently $T$ is also a homomorphism. In fact, even if $T$ is not unital, the same argument works (modulo a few technical details) to show that: 
Theorem 2.1 ([6], 7], [8], [18] and B.1 in [10]). Suppose that $T: A \rightarrow B$ is a linear map between approximately unital operator algebras. Then $T$ is a surjective complete isometry if and only if $T=u \theta(\cdot)$, where $u$ is a unitary in the diagonal $\Delta(M(B))$ of the multiplier algebra of $B$, and where $\theta: A \rightarrow B$ is a surjective completely isometric homomorphism which is a restriction of a ${ }^{*}$-isomorphism between the $C^{*}$-envelopes of $A$ and $B$. Furthermore, the surjective complete isometry $T$ is unital if and only if the $u$ above equals $1_{B}$, and if and only if $T$ is a homomorphism.

In the remainder of this section, and in the following section, we attempt to find analogous results for nonsurjective complete isometries between non-selfadjoint operator algebras, and we give some applications. These will generalize Theorem 1.1, stated in the introduction. The first set of characterizations will be collected in the following theorem. We apologize in advance to the reader - since this is quite similar to the analogous result for selfadjoint operator algebras (Theorem 3.1 in [12]), we cannot justify repeating all the ideas and notation established there. Thus we must refer the reader to that paper for further details regarding our notation and proof.

Suppose that $B$ is a subalgebra of a $C^{*}$-algebra $C$. A reducing $\wedge$-compression of a map $T: X \rightarrow B$ is a map $R: X \rightarrow C^{\star \star}$ such that there exist projections $e, f \in C^{\star \star}$ such that

$$
R=\widehat{T(\cdot)} e=f \widehat{T(\cdot)}=f \widehat{T(\cdot)} e .
$$

Thus if $C$ is represented on its universal Hilbert space $H_{u}$, then there are subspaces $L, M$ of $H_{u}$ (corresponding to $e, f$ ) such that $R=P_{M} T(\cdot)_{L}$, and $T=R \oplus S$ for a completely contractive $S: X \rightarrow B\left(L^{\perp}, M^{\perp}\right)$.

Theorem 2.2. Let $T: A \rightarrow B$ be a linear completely contractive map between approximately unital operator algebras. Suppose that $B$ is a subalgebra of a $C^{*}$ algebra $C$ (for example, $C=C_{e}^{*}(B)$ ). Then the following are equivalent:

(i) $T$ is a complete isometry.

(ii) There are a left ideal $J$ and a right ideal $K$ of $C$ such that $q_{J+K} \circ T$ is the restriction to $A$ of a linear map $S: C_{e}^{*}(A) \rightarrow C /(J+K)$ such that $S$ composed with the canonical injection $C /(J+K) \rightarrow(1-q) C^{\star \star}(1-p)$ is a triple morphism. (Here $q$ and $p$ are the so-called support projections of $J$ and $K$ respectively. See 12 for the details.)

(iii) $T$ has a reducing $\wedge$-compression $R: A \rightarrow C^{\star \star}$ which is the restriction of a 1-1 triple morphism from $C_{e}^{*}(A)$ into $C^{\star \star}$.

(iv) There are a partial isometry $u$ in $C^{\star \star}$, a projection $p$ in $C^{\star \star}$ (which may be chosen so that $1-p$ is the initial projection $u^{*} u$ of $u$ ), and a 1-1 *-homomorphism $\theta: C_{e}^{*}(A) \rightarrow(1-p) C^{\star \star}(1-p)$, such that

$$
\left.\widehat{T(a)}(1-p)=u \theta(a) \quad \text { and } \quad \theta(a)=u^{*} \widehat{T(a)}\right)
$$

for all $a \in A$. If $A$ is unital, then $\theta(1)=u^{*} u$.

If $A$ is unital, these are equivalent to:

(v) There exists a closed left ideal $J$ of $C$ such that for all $a \in A$ we have

$$
q_{J}(T(a))=u \pi(a) \text { and } \pi(a)=u^{*} q_{J}(T(a)) \text {, }
$$

where $q_{J}: C \rightarrow C / J$ is the canonical quotient map, $\pi: A \rightarrow C /\left(J+J^{*}\right)$ is a completely isometric homomorphism which is the restriction of a 1-1 *-homomorphism $C_{e}^{*}(A) \rightarrow M_{00}\left(C /\left(J+J^{*}\right)\right)$ (see [12] for definitions), and 
$u$ is a partial isometry in $B / J \subset C / J$ which may be taken to be $u=$ $q_{J}(T(1))$.

(vi) There exist a $C^{*}$-subalgebra $D$ of $C$ containing $T(A)^{*} T(A)$, a closed twosided ideal $I$ in $D$, and a surjective ${ }^{*}$-isomorphism $\pi: C_{e}^{*}(A) \rightarrow D / I$, such that

$$
q_{N} \circ T=U \pi(\cdot)
$$

where $q_{N}$ is the canonical quotient triple morphism from the triple subsystem $Z=T(A) D$ of $C$ onto the quotient of $Z$ by the triple ideal $N=Z I$, and $U$ is a unitary in the triple system $Z / N$. One may take $U=q_{N}(T(1))$.

Proof. This is very similar to the proof of 3.1 in 12 . So we will be brief and omit most of the details. In particular, it is a simple exercise that any of (ii)-(vi) implies (i). Let $Z$ be the triple system in $C$ generated by $T(A)$. In [10, section 4 (see also [55]), it is shown that $C_{e}^{*}(A)$ is a triple envelope of $A$. Thus, by the universal property of this envelope mentioned in the introduction, there exists a surjective triple morphism $\rho: Z \rightarrow C_{e}^{*}(A)$ such that $\rho(T(a))=a$ for all $a \in A$. Set $N=\operatorname{Ker} \rho$, and obtain a surjective triple isomorphism $\xi: C_{e}^{*}(A) \rightarrow Z / N$. Appealing to Lemma 2.10 in 12 almost immediately gives (vi). We may then follow the proof of (i) $\Rightarrow$ (iii) of 3.1 in [12, replacing $B$ there by $C$, and so on, to obtain a completely isometric partial triple morphism $\theta$ of $C_{e}^{*}(A)$ into $C /(J+K)$. The restriction of $\theta$ to $A$ is $q_{J+K} \circ T$. This gives (ii). We also obtain, as in 3.1 of [12], a 1-1 triple morphism $\tilde{T}: C_{e}^{*}(A) \rightarrow C^{\star \star}(1-p)$ taking $a \in A$ to $\widehat{T(a)}(1-p)=(1-q) \widehat{T(a)}(1-p)=(1-q) \widehat{T(a)}$. This gives (iii). The proofs of (iv) and (v) are then similar to the matching items of (vi) in 3.1 of [12. We will simply prove the assertions in (iv) that were not in the original version of [12. Note that the partial isometry $u$ coming from the proof of 3.1 in [12] may be defined to be the weak* limit of $T\left(e_{\alpha}\right)(1-p)$, for a c.a.i. $\left\{e_{\alpha}\right\}$ of $A$. Thus the projection

$$
u^{*} u=\mathrm{w}^{*} \lim _{\alpha} u^{*} T\left(e_{\alpha}\right)(1-p)=\mathrm{w}^{*} \lim _{\alpha} \theta\left(e_{\alpha}\right)
$$

satisfies $u^{*} u(1-p)=u^{*} u$, and is therefore dominated by $1-p$. By the relation involving $p$ and $q$ above, it also follows that

$$
u^{*} T(a)=\mathrm{w}^{*} \lim _{\alpha}(1-p) T\left(e_{\alpha}\right)^{*} T(a)=\mathrm{w}^{*} \lim _{\alpha}(1-p) T\left(e_{\alpha}\right)^{*} T(a)(1-p),
$$

which is the formula given in 12 for the homomorphism $\theta$ we are looking for. Also, by the second last displayed formula above, we have $u^{*} u \theta(a)=\lim _{\alpha} \theta\left(e_{\alpha} a\right)=\theta(a)$, and similarly for $\theta(a) u^{*} u$.

Remarks. 1). If $A$ and $B$ are unital, another route to the proof of some parts of the theorem may be given via first extending $T$ to a complete isometry on the Paulsen system [56. However, this approach does not seem to readily give all we need. It does show that the projection $p$ in (iv) may be chosen to be an open projection.

2 ). We sketch how 1.1 follows from (v) of our theorem. In particular, as stated in the introduction, under the hypotheses of 1.1, $T$ is completely isometric and we may apply 2.2. Since we find ourselves in a commutative context in 1.1, the (now two-sided) ideal $J=J+J^{*}$ corresponds to a set of continuous functions vanishing on some closed subset $E^{\prime}$ of $\partial B$. Thus $C /\left(J+J^{*}\right)=C(\partial B) / J$ is then a copy of $C\left(E^{\prime}\right)$. If $\pi$ is the 1-1 $*$-homomorphism from $C(\partial A)$ into $M_{00}\left(C /\left(J+J^{*}\right)\right)=C\left(E^{\prime}\right)$, let $E$ be the clopen subset $\operatorname{Ker} \pi(1)$ of $E^{\prime}$. If $y \in E$ and $\epsilon_{y}$ is 'evaluation at $y$ ', then $\pi^{*}\left(\epsilon_{y}\right)$ is a character on $C(\partial A)$, and is therefore a point evaluation at a point 
$\varphi(y) \in \partial A$. The map $\varphi: E \rightarrow \partial A$ is then easily seen to be a continuous surjection. Item (v) of the above theorem then gives

$$
T(f)(y)=\gamma(y) f(\varphi(y)) \text { and } \quad f(\varphi(y))=\overline{\gamma(y)} T(f)(y)
$$

for all $y \in E$ and $f \in A$. Here $\gamma: E \rightarrow\{0\} \cup \mathbb{T}$ is the partial isometry $u$ viewed as a function on $E$. Thus $1=\overline{\gamma(y)} T(1)(y)$, so that $\gamma$ in fact maps into $\mathbb{T}$.

In the proof of 2.2, we obtained projections $p, q$ in $C^{\star \star}$ such that

$$
\widehat{T(\cdot)}(1-p)=(1-q) \widehat{T(\cdot)}=(1-q) \widehat{T(\cdot)}(1-p),
$$

and saw that this expression is the restriction to $A$ of a 1-1 triple morphism $\tau$ : $C_{e}^{*}(A) \rightarrow(1-q) C^{\star \star}(1-p)$. We call such $p$ accomplishing all this a reducing projection for $T$. From the above it is clear that

$$
T(a b)(1-p)=T(a) T(1)^{*} T(b)(1-p)
$$

for all $a, b \in A$. We will use this fact later on. We remark that in the light of the work in [16] on isometries (as opposed to complete isometries), it seems plausible that there is an extremal reducing projection for $T$, and that one might want to use this one in applications. In the applications given later in our paper, any reducing projection will suffice.

Corollary 2.3. Let $T: A \rightarrow B$ be a unital completely contractive linear map between unital operator algebras. Suppose that $B$ is a unital subalgebra of a unital $C^{*}$-algebra $C$. Then saying that $T$ is a complete isometry is equivalent to any one of (ii)-(vi) in the previous theorem, but with the following changes: One may omit all mention of $u$, change 'triple morphism' to '*-homomorphism', and $K$ to $J^{*}$, in (ii), and note that the expression $\widehat{T(a)}(1-p)$ in (ii) and (iv) now coincides with $(1-p) \widehat{T(a)}(1-p)$. One may change (vi) to read: there exist a $C^{*}$-subalgebra $D$ of $C$ containing $T(A)$, a closed two-sided ideal $I$ in $D$, and a surjective ${ }^{*}$-isomorphism $\pi: C_{e}^{*}(A) \rightarrow D / I$, such that $q_{I} \circ T=\pi$.

We note in passing that 2.3 is a much simpler result than 2.2 and is closely related to [15, Theorem 4.1. Appropriate parts of this result may also be stated in terms of block diagonal matrices as in the introduction. Suppose for example that $T: A \rightarrow B$ is a unital complete isometry between unital operator algebras, and suppose that $B$ is a unital subalgebra of a unital $C^{*}$-algebra $C$. Let $H_{u}$ be the Hilbert space of the universal representation of $C$; thus we have ${ }^{*}$-subalgebras $C \subset C^{\star \star} \subset B\left(H_{u}\right)$. Then, corresponding to the projection $p$, there is a subspace $K$ of $H_{u}$ such that $P_{K} T(\cdot)=T(\cdot) P_{K}=P_{K} T(\cdot) P_{K}$, and if we define $R: A \rightarrow B(K)$ by these equal expressions, then $R$ is a completely isometric homomorphism (and $R$ is also the restriction of a 1-1*-homomorphism defined on $\left.C_{e}^{*}(A)\right)$. Then $T=R \oplus S$ for a completely contractive unital map $S$, as in the introduction. A similar remark holds in connection with Theorem [2.2 we will see a concrete exhibition of this momentarily in 2.5.

We proceed with some typical applications of 2.2. The first is a special case worthy of consideration (for the proof one need only note that the simplicity requirement ensures that $I=\{0\}$ in [2.3):

Corollary 2.4. Let $T: A \rightarrow B$ be a unital complete isometry between unital operator algebras $A$ and $B$. Suppose that $B$ is a unital subalgebra of a $C^{*}$-algebra $C$, and suppose further that the $C^{*}$-subalgebra of $C$ generated by $T(A)$ is simple 
(for example, if $T$ is a map into $M_{n}$ whose range generates $M_{n}$ ). Then $T$ is automatically a homomorphism, indeed the restriction of a 1-1 *-homomorphism $C_{e}^{*}(A) \rightarrow C$.

Corollary 2.5. Let $T: A \rightarrow B$ be a linear complete isometry from a unital operator algebra into a finite-dimensional $C^{*}$-algebra.

(1) There exist a $C^{*}$-subalgebra $C$ of $B$, a unital ${ }^{*}$-homomorphism $\pi: C_{e}^{*}(A) \rightarrow$ $C$, a partial isometry $u$ in $B$ with initial projection e equal to the identity of $C$, and a final projection $f$, such that $T(\cdot) e=f T(\cdot)=u \pi(\cdot)$.

(2) $A$ is completely isometrically homomorphic to a subalgebra of $B$.

(3) If $B=M_{m}$, then there exists a unital 1-1 *-homomorphism $\theta: C_{e}^{*}(A) \rightarrow$ $M_{n}$ for an integer $n \leq m$, and there are unitary $m \times m$ matrices $U$ and $V$, and a completely contractive map $S: A \rightarrow M_{m-n}$, with $T(a)=$ $U \operatorname{diag}\{\theta(a), S(a)\} V$ for all $a \in A$.

Proof. (1) By the last assertion in 2.2 there exist projections $e, f \in B$, a 1-1 unital *-homomorphism $\pi: C_{e}^{*}(A) \rightarrow e B e$, and a partial isometry $u$ such that the desired properties hold. Note that $u u^{*} T(\cdot)=u \pi(\cdot)=T(\cdot) e$. (2) follows immediately from (1). So does (3): choose an orthonormal basis $B$ for Ran $e$, contained in an orthonormal basis for $\mathbb{C}^{m}$. Choose another orthonormal basis for $\mathbb{C}^{m}$ containing $u(B)$. The matrix of $T(a)$ with respect to these two orthonormal bases is easily seen to be $\operatorname{diag}\{\theta(a), S(a)\}$, for maps $\theta, S$ of the specified form.

Note that (3) above implies that $C_{e}^{*}(A)$ is a finite-dimensional $C^{*}$-algebra, and consequently *-isomorphic to a finite direct sum of blocks $M_{n_{k}}$. Thus the $\theta$ in (3) is a direct sum of finite multiples of the identity representation of the $M_{n_{k}}$. This allows one to simplify the characterization still further. For example, if $T_{n}$ is the upper triangular $n \times n$ matrices, then by the above, and because we know that $C_{e}^{*}\left(T_{n}\right)=M_{n}$, we may deduce that a linear map $\varphi: T_{n} \rightarrow M_{m}$ is a complete isometry if and only if there exist unitary matrices $U$ and $V$ of appropriate sizes, and a completely contractive map $S: T_{n} \rightarrow M_{m-n}$, such that $\varphi(A)=U \operatorname{diag}\{A, S(A)\} V$ for all $A \in T_{n}$. This example can no doubt also be deduced from the very general results on isometries between nest algebras from [1].

The ideas in the last few results also illustrate the method for computing the $C^{*}$-envelope (which in this case equals the injective envelope) of a unital subspace $A$ of $M_{n}$. Namely, first one replaces $M_{n}$ by the unital $*$-subalgebra $B$ generated by $A$. By the abstract principles used above, there is a central projection $p$ in $B$ with $B(1-p)=C_{e}^{*}(A)$. Since $B$ is unitarily equivalent to (up to multiplicity) a direct sum $\bigoplus_{k} M_{n_{k}}$ of full matrix algebras $M_{n_{k}}$, the central projection $p$ is unitarily equivalent to a direct sum of identity matrices $I_{n_{i}}$ or $0_{n_{i}}$ matrices. Thus $C_{e}^{*}(A)$ is simply a direct sum of a subcollection of the $M_{n_{k}}$ above. A similar idea works for computing the triple or injective envelope of a subspace of $M_{n}$, except that now we have two projections $p$ and $q$. It is instructive, and is also a good source of counterexamples, to select a set of two or three matrices in $M_{n}$, and then to compute the $C^{*}$-envelope or triple envelope of their span.

We close this section with a related fact clarifying the relationship between the categories of unital operator spaces and approximately unital operator algebra:

Corollary 2.6. If $A$ is an approximately unital operator algebra, and if $A$ is linearly completely isometric to a unital operator space, then $A$ is a unital algebra. 
Proof. If $V$ is the unital operator space, then it follows that the triple envelope of $A$ is triple isomorphic to the triple envelope of $V$, via a morphism taking $V$ onto $A$. The latter envelope may be taken to be $C_{e}^{*}(V)$, which is unital [22]. Also in [10] we showed that the triple envelope of an approximately unital operator algebra $A$ may be taken to be $C_{e}^{*}(A)$ (part of this was observed in [55, too). It is now easy to see (using 2.10 in [12, for example) that $C_{e}^{*}(A)$ is unital, whence $A$ is also unital.

\section{Characterizations of particular Classes of isometries}

If one considers Theorem 1.1 a 'nicest class' of isometries suggests itself: namely those for which $E=\partial A$. A moment's thought (using Lemma 2.10 of [12], for example) shows that this class falls within the class of maps $T: A \rightarrow B$ which are the restrictions of a 1-1 triple morphism $C(\partial A) \rightarrow C(\partial B)$. We shall call these the Shilov isometries. More generally, if $A$ and $B$ are approximately unital operator algebras, we say that a map $T: A \rightarrow B$ is a Shilov isometry if $T$ is the restriction to $A$ of a 1-1 triple morphism $C_{e}^{*}(A) \rightarrow C_{e}^{*}(B)$.

The following two results, which are fairly superficial, we state to afford a comparison with subsequent results.

Lemma 3.1. Let $T: A \rightarrow B$ be a linear map between unital $C^{*}$-algebras. The following are equivalent:

(i) $T$ is a Shilov isometry (that is, a 1-1 triple morphism).

(ii) $T=u \pi(\cdot)$ for a 1-1 *-homomorphism $\pi: A \rightarrow B$ and a partial isometry $u \in B$ such that $u^{*} u=\pi\left(1_{A}\right)$.

(iii) $T$ is a complete isometry with $\{0\}=J \subset C=B$ in Theorem 2.9 (v).

We may take $u=T\left(1_{A}\right)$. If these equivalent conditions hold, then $T(A)$ contains a unitary (resp. isometry, coisometry) of $B$ if and only if $u=T\left(1_{A}\right)$ is a unitary (resp. isometry, coisometry) of $B$. Indeed, if $1_{B} \in T(A)$, then $T\left(1_{A}\right)$ is a unitary of $B$ and $T(A)=\pi(A)$, so that $\operatorname{Ran} T$ is a $C^{*}$-subalgebra of $B$, and also $\pi\left(1_{A}\right)=1_{B}$. If in addition $T\left(1_{A}\right)=1_{B}$, then $T$ is a ${ }^{*}$-homomorphism.

Proof. The equivalence of (i)-(iii) we leave as an exercise (using Lemma 2.10 in [12], for example). Set $u=T(1)$. We will use the fact that if $R, S$ are contractions between Hilbert spaces with $R S=I$, then $S$ is an isometry and $R=S^{*}$. Thus if $T(A)$ contains a coisometry $v=T\left(a_{0}\right)$, then $v=u \pi\left(a_{0}\right)$, giving $1_{B}=u\left(\pi\left(a_{0}\right) v^{*}\right)$. By the fact above about Hilbert space operators, $u$ is a coisometry. By symmetry we obtain the assertions for isometries and unitaries.

If $1_{B} \in T(A)$, then $u$ is a unitary of $B$ by the above. Since $T$ is a triple morphism, it is evident that $\operatorname{Ran} T$ is a $C^{*}$-subalgebra of $B$. Write $1_{B}=T\left(a_{0}\right)$. Then $\pi\left(a_{0}\right)=u^{*} u \pi\left(a_{0}\right)=u^{*} T\left(a_{0}\right)=u^{*}$, so that $u^{*}$ and $u$ are in the $C^{*}$-algebra $\pi(A)$. Hence $T(A)=u \pi(A)=\pi(A)$. Also $\pi\left(1_{A}\right)=u^{*} T(1)=1_{B}$, since $u$ is unitary.

Lemma 3.2. Let $T: A \rightarrow B$ be a linear map between unital operator algebras. The following are equivalent:

(i) $T$ is a Shilov isometry.

(ii) $T=u \pi(\cdot)$ for a completely isometric homomorphism $\pi$ on $A$ which is the restriction of a $1-1^{*}{ }_{-}$homomorphism $C_{e}^{*}(A) \rightarrow C_{e}^{*}(B)$, and a partial isometry $u \in B \subset C_{e}^{*}(B)$ with $u^{*} u=\pi\left(1_{A}\right)$.

(iii) $T$ is a complete isometry with $\{0\}=J \subset C=C_{e}^{*}(B)$ in Theorem 2.2 (v). 
We may take $u=T\left(1_{A}\right)$. If these equivalent conditions hold, then $T(A)$ contains a unitary (resp. isometry, coisometry) of $C_{e}^{*}(B)$ if and only if $u=T\left(1_{A}\right)$ is a unitary (resp. isometry, coisometry) of $C_{e}^{*}(B)$. Indeed, if $1_{B} \in T(A)$, then $\pi(A) \subset$ $B$, and also $\pi\left(1_{A}\right)=1_{B}$. If in addition $T\left(1_{A}\right)=1_{B}$, then $T$ is a homomorphism (namely $T=\pi$ ).

Proof. Again we leave the equivalences (i)-(iii) as an exercise. If $1_{B} \in T(A)$, then $1_{B} \in \tilde{T}(A)$, where $\tilde{T}$ is the triple morphism $C_{e}^{*}(A) \rightarrow C_{e}^{*}(B)$ extending $T$. Applying 3.1. we see that $u=T(1)$ is a unitary of $C_{e}^{*}(B)$. Selecting $a_{0} \in A$ such that $T\left(a_{0}\right)=1_{B}$, it follows as in the proof of Lemma 3.1 that $u^{*}=\pi\left(a_{0}\right) \in \pi(A)$. Notice that

$$
u T\left(a_{0}^{2}\right)=u^{2} \pi\left(a_{0}^{2}\right)=u T\left(a_{0}\right) \pi\left(a_{0}\right)=u \pi\left(a_{0}\right)=T\left(a_{0}\right)=1_{B} .
$$

Thus $u^{*}=T\left(a_{0}^{2}\right)$, whence $\pi(A)=u^{*} T(A) \in B^{2} \subset B$. The other assertions are left as an exercise.

If we drop the hypothesis that $T$ is a Shilov isometry, then things become less simple. The problem is that our abstract result, Theorem [2.2, is difficult to use if the 'Shilov boundaries' are complicated, as is the case with $H^{\infty}(\mathbb{D})$ say. There is, however, a class of complete isometries which are not Shilov isometries, but which are fairly tractable. Namely, following Matheson [36], we say that a (not necessarily surjective) linear complete isometry $T: A \rightarrow B$ between unital operator algebras is left type 1 if $B \cap J=\{0\}$ for the left ideal $J$ in Theorem 2.2 (v). Equivalently, $T$ is left type 1 if the restriction to $B$ of the canonical quotient map $C_{e}^{*}(B) \rightarrow C_{e}^{*}(B) / J$ is 1-1; or (using the fact that $p \in J^{* *}=B^{* *} p$ from the proof of Lemma 2.8 in [12]) equivalently if the map $b \mapsto b(1-p)$ on $B$ is $1-1$, where $p$ is as in the proof of Theorem 2.2 In fact, it is the last condition that will be mostly used below; thus one might want to define 'left type 1 ' as this condition, for some reducing projection $p$ for $T$ (see the definition and discussion just above Corollary 2.3).

We remark that if one replaces $C_{e}^{*}(B)$ by any $C^{*}$-algebra containing $B$, most of our proofs below will still go through. In the corollaries below we will however regard $B$ as a unital subalgebra of $C_{e}^{*}(B)$, and any adjoints $b^{*}$ of elements in $B$ are taken in that $C^{*}$-algebra.

There is an analogous definition of right type 1 , namely that $b \mapsto(1-q) b$ is $1-1$ on $B$, where $q$ is the projection discussed in and after the proof of Theorem 2.2 One can show that $T$ is right type 1 if and only if the map $T^{*}: A^{*} \rightarrow B^{*}$ given by $T^{*}\left(a^{*}\right)=T(a)^{*}$ is left type 1 . We say that $T$ is type 1 if it is both left and right type 1 .

Note that if the function algebras in Theorem 1.1 are $C(K)$ spaces, then 'most' isometries are type 2 (non-type 1 ) isometries (since the type 1 isometries between $C(K)$ spaces are exactly the maps characterized in Lemma 3.1. Quite obviously, any Shilov isometry between function or operator algebras is type 1, since in this case $J=\{0\}$. However, it is easy to find type 1 isometries that are not Shilov isometries. For example, Alec Matheson showed us an interesting class of examples constructed along the following lines. Let $A=A(\mathbb{D})$ be the disk algebra, and let $\psi$ be the Riemann map from the closed disk onto the upper half of the closed disk. Then let $\varphi(z)=\psi(z)^{2}$, and define $T f(z)=f(\varphi(z))$ for $f \in A$ and $z$ in the closed unit disk. It is easy to see that $T: A \rightarrow A$ is a unital type 1 isometry (it is type 1 because of the well-known fact that a nontrivial function in $A(\mathbb{D})$ cannot vanish on a nontrivial arc of the circle) that is not a Shilov isometry. 
For us, the main interest in type 1 isometries is the following point: Ideally one would like to classify isometries in terms of composition operators. But the question of what we mean by the term 'composition' needs to be clarified. For example, classical $H^{\infty}(\mathbb{D})$ lives inside $C\left(\partial H^{\infty}\right)=L^{\infty}(\mathbb{T})$, and hence if in this context we want to describe isometries in terms of composition operators, we need to specify composition by what? For a result to earn the title of a true $H^{\infty}$ result, such composition should be described in terms of point transformations on $\mathbb{D}$ rather than $\partial H^{\infty}$ or $\mathbb{T}$. The papers [36], 20] suggest that for common function algebras (on domains in $\mathbb{C}$, for example), it is the type 1 isometries between these algebras which seem to have some hope of being classifiable as composition operators on their domains (as opposed to on their Shilov boundaries). What makes this work in [36] is the commutative analogue of our next result (see also the remarks at the end of this section for a demonstration of this technique of Matheson in a concrete case). Thus our next result should be useful in the study of particular noncommutative operator algebras.

Corollary 3.3. Let $T: A \rightarrow B$ be a left type 1 linear complete isometry between unital operator algebras such that $T(1)$ commutes with $T(a)$ for all $a \in A$. Then

$$
T(1) T(a b)=T(a) T(b)
$$

for all $a, b \in A$. Therefore if, further, $T(1)$ is invertible, then we may write

$$
T=T(1) \theta(\cdot)
$$

for a unital 1-1 'completely bicontinuous' homomorphism $\theta$ defined on A, namely $\theta=T(1)^{-1} T(\cdot)$.

Proof. It suffices to show that $T(1) T(a b)(1-p)=T(a) T(b)(1-p)$. We obtain using $\left(^{*}\right)$ (see the comment following Theorem 2.2) that

$$
T(1) T(a b)(1-p)=T(1) T(a) T(1)^{*} T(b)(1-p) .
$$

Again using $(*)$, and the fact that $T(1)$ commutes with $T(a)$, we get

$$
T(1) T(a b)(1-p)=T(a) T(1) T(1)^{*} T(b)(1-p)=T(a) T(b)(1-p) .
$$

To see the final assertion, note that in the noncommutative case

$$
T(1)^{-1} T(a) T(1)^{-1} T(b)=T(1)^{-2} T(a) T(1) T(1)^{-1} T(b)=T(1)^{-1} T(a b),
$$

since $T(a b)=T(1)^{-1} T(a) T(b)$.

Corollary 3.4. Let $T: A \rightarrow B$ be a left type 1 unital linear complete isometry between unital operator algebras. Then $T$ is a homomorphism.

Corollary 3.5. Let $T: A \rightarrow B$ be a left type 1 linear complete isometry between unital operator algebras. Suppose that $T\left(1_{A}\right) \in \Delta(B)$. Then $u=T\left(1_{A}\right)$ is a partial isometry in $C_{e}^{*}(B)$, and there exists a completely isometric homomorphism $\theta: A \rightarrow$ $B$ such that

$$
T=u \theta(\cdot) \quad \text { and } \quad \theta=u^{*} T(\cdot) .
$$

Proof. If we define $\theta=u^{*} T(\cdot)$, then $\theta: A \rightarrow B$. Also,

$$
u \theta(a)(1-p)=u u^{*} T(a)(1-p)=T(a)(1-p)
$$

by $\left(^{*}\right)$. Since we are in the left type 1 situation, the last equation implies that $T=u \theta(\cdot)$. Similar considerations show that $\theta$ is a homomorphism. Similarly, we 
may also conclude from $\left(^{*}\right)$ and the left type 1 hypothesis that $u u^{*} u=u$, so that $u$ is a partial isometry in $C_{e}^{*}(B)$. Then $\theta$ is a complete isometry, since for example

$$
\|a\|=\|T(a)\| \geq\left\|u^{*} T(a)\right\|=\|\theta(a)\| \geq\|u \theta(a)\|=\|T(a)\|=\|a\| .
$$

Corollary 3.6. Let $T: A \rightarrow B$ be a left type 1 linear complete isometry between unital operator algebras. Suppose that $1_{B} \in \operatorname{Ran} T$. Then $u=T\left(1_{A}\right)$ is a coisometry with $u, u^{*} \in B$ (that is, $u \in \Delta(B)$ ), and there exists a completely isometric homomorphism $\theta: A \rightarrow B$ possessing all the properties of the previous corollary. Also $u^{*} \in \theta(A)$. If, further, $T\left(1_{A}\right)$ commutes with $T(A)$ (or even simply with $T\left(a_{0}^{2}\right)$, where $\left.T\left(a_{0}\right)=1_{B}\right)$, or if $T$ is also right type 1 , then $u=T(1)$ is a unitary of $\Delta(B)$, and $\theta\left(1_{A}\right)=1_{B}$.

Proof. If $T\left(a_{0}\right)=1_{B}$, then we have $\left\|a_{0}\right\|=1$. As in the first displayed equation in the proof of Corollary 3.3 (with $a=b=a_{0}$ ), we have from $(*)$ that

$$
u T\left(a_{0}^{2}\right)(1-p)=u T\left(a_{0}\right) u^{*} T\left(a_{0}\right)(1-p)=u u^{*} T\left(a_{0}\right)(1-p)=T\left(a_{0}\right)(1-p) .
$$

Thus $T(1) T\left(a_{0}^{2}\right)=T\left(a_{0}\right)=1_{B}$. Since $T(1)$ and $T\left(a_{0}^{2}\right)$ are contractions on some Hilbert space, this forces $u=T(1)$ to be a coisometry, and $T\left(a_{0}^{2}\right)=T(1)^{*}$. Thus $T(1)^{*} \in B$, and we may apply Corollary 3.5. We have $\theta\left(a_{0}\right)=u^{*} T\left(a_{0}\right)=u^{*}$.

If $u=T(1)$ commutes with $T\left(a_{0}^{2}\right)=u^{*}$, then it is clear that $u$ is a unitary. Similarly, if $T$ is both 'left-' and 'right type 1', then an argument symmetric to the above shows that $u$ is an isometry:

$(1-q) T\left(a_{0}^{2}\right) T(1)=(1-q) T\left(a_{0}\right) u^{*} T\left(a_{0}\right) u=(1-q) T\left(a_{0}\right) u^{*} u=(1-q) T\left(a_{0}\right)=(1-q)$.

Thus $T\left(a_{0}^{2}\right) T(1)=1_{B}$, so that $u$ is an isometry, and $u$ is unitary.

Returning to Corollary [3.3, we note that if, in addition to the hypotheses there, $B$ is a commutative algebra and $T(1)$ is invertible in $B$, then the homomorphism $\theta$ in that corollary maps into $B$, so that $A$ is commutative too. If, in addition, $B$ is a function algebra, then from Gelfand theory it follows that the homomorphism $\theta$ in that corollary is a contraction. Consequently it is an isometry, since

$$
\|\theta(a)\| \geq\|T(1) \theta(a)\|=\|T(a)\|=\|a\| .
$$

Corollary 3.7. Let $T: A \rightarrow B$ be a left type 1 linear complete isometry between unital operator algebras, and suppose that $B$ is commutative. If $T(A)$ does not vanish identically at any point of the maximal ideal space of $B$, then $A$ is commutative, $T(1)$ is invertible in $B$, and

$$
T=T(1) \theta(\cdot)
$$

for a unital completely bicontinuous homomorphism $\theta: A \rightarrow B$.

Proof. If $\chi$ is a character of $B$ with $\chi(T(1))=0$, then by Corollary 3.3 it follows that $\chi\left(T(a)^{2}\right)=\chi(T(1)) \chi\left(T\left(a^{2}\right)\right)=0$ for all $a \in A$. Thus $\chi(T(A))=0$, which is a contradiction. By Gelfand theory, $T(1)$ is invertible in $B$. The rest follows from the remark before the corollary, and Corollary 3.3.

Corollary 3.8. Let $T: A \rightarrow B$ be a type 1 linear isometry between unital function algebras, and suppose that $B$ is a closed unital subalgebra of $C(K)$ for a compact Hausdorff space $K$. If $T(A)$ does not vanish identically at any point of $K$, then $T(1)$ is nonzero at every point in $K$, and

$$
T=T(1) \theta(\cdot)
$$


for a unital isometric homomorphism $\theta: A \rightarrow C(K)$.

Proof. The argument in the previous corollary shows that $u=T(1)$ is nonzero on $K$, and hence invertible in $C(K)$. The rest follows from the remark before Corollary 3.7 .

There are surely noncommutative versions of the previous two corollaries. One such result goes as follows:

Corollary 3.9 (cf. 39]). Let $T: A \rightarrow B$ be a left type 1 linear complete isometry between unital operator algebras such that $T(1)$ commutes with $T(a)$ for all $a \in A$. Suppose that $B$ is a unital subalgebra of $B(H)$, suppose that $T(A) H$ is dense in $H$, and suppose that there is a constant $K>0$ such that for all $\zeta \in H$,

$$
\|\zeta\| \leq K \sup \{\|T(a) \zeta\|: a \in \operatorname{Ball}(A)\} .
$$

Then $T(1)$ is an invertible operator on $H$, and we may write

$$
T=T(1) \theta(\cdot)
$$

for a unital 'completely bicontinuous' homomorphism $\theta: A \rightarrow B(H)$. If indeed $K=1$, then $T(1)$ is unitary and $\theta$ is completely isometric.

Proof. For the first part we need only show that $T(1)$ is invertible, by Corollary 3.3. We have

$$
\|\zeta\| \leq K^{2} \sup _{b \in \operatorname{Ball}(A)} \sup _{a \in \operatorname{Ball}(A)}\{\|T(a) T(b) \zeta\|\} \leq K^{2}\|T(1) \zeta\|,
$$

using the fact that $T(a) T(b)=T(a b) T(1)$ from Corollary 3.3 Thus $T(1)$ is bicontinuous and has closed range. Moreover, if $K=1$, then $T$ is isometric. However,

$$
T(A) H=T(A) \overline{T(A) H} \subset \overline{T(A) T(A) H} \subset \overline{T(1) H}
$$

(by continuity and the fact that $T(a) T(b)=T(1) T(a b)$ ). So $T(1)$ maps onto $H$. Thus $T(1)$ is invertible. We leave the rest to the reader.

We end Part I by describing very briefly some results found together with Alec Matheson. These are applications of the ideas above, in an attempt to gain insight into the difficult open problems concerning isometries $T: A \rightarrow A$, where $A=$ $H^{\infty}(\mathbb{D})$ and $\mathbb{D}$ is the open unit disk. We first remark that it is easy to characterize contractive homomorphisms of $H^{\infty}(\mathbb{D})$, by the methods of p. 144 in 25 . These are exactly the composition operators $T(f)=f \circ \tau$ on $\mathbb{D}$, for a $\tau \in \operatorname{Ball}\left(H^{\infty}(\mathbb{D})\right)$. If $T$ is also an isometry, then one can say quite a bit more about $\tau$. Then, using the ideas above, one can show that a general type 1 isometry $T: H^{\infty}(\mathbb{D}) \rightarrow H^{\infty}(\mathbb{D})$ is of the form $T(f)=T(1) f \circ \tau$ on $\mathbb{D}$, for all $f \in H^{\infty}(\mathbb{D})$, and where $\tau$ is as above (i.e., the map $f \mapsto f \circ \tau$ is an isometric homomorphism on $H^{\infty}(\mathbb{D})$ ).

Leaving the type 1 case, and considering general unital isometries $T: H^{\infty}(\mathbb{D}) \rightarrow$ $H^{\infty}(\mathbb{D})$, we note first that because the injective envelope and triple envelope of $H^{\infty}(\mathbb{D})$ coincide with $L^{\infty}(\mathbb{T})$ (see the Remark before Definition 5.5below), any such $T$ extends to a unital isometry $\tilde{T}: L^{\infty}(\mathbb{T}) \rightarrow L^{\infty}(\mathbb{T})$. We obtain from Theorem 1.1 a closed ideal $I$ in $L^{\infty}(\mathbb{T})$, as in that theorem. We let $J=H^{\infty}(\mathbb{D}) \cap I$. Denoting by $q_{I}$ and $q_{J}$ respectively the canonical quotient maps $L^{\infty}(\mathbb{T}) \rightarrow L^{\infty}(\mathbb{T}) / I$ and $H^{\infty}(\mathbb{D}) \rightarrow H^{\infty}(\mathbb{D}) / J$, we have that $q_{J} \circ T$ is an isometric homomorphism, and $q_{I} \circ \tilde{T}$ is a $1-1 *$-homomorphism (all this in fact is true in much greater generality). Let $\imath$ and $\tau$ respectively denote the functions $\imath: z \rightarrow z$ and $T(\imath)$ on $\mathbb{D}$. Then $\tau=T(\imath)$ 
is a self-map on $\mathbb{D}$ which induces a composition operator $C_{\tau}: H^{\infty} \rightarrow H^{\infty}: f \mapsto$ $f \circ \tau$, which approximates the action of $T$ modulo the ideal $J$ in many senses. For example, $q_{J} \circ C_{\tau}$ agrees with $q_{J} \circ T$ on the disk algebra. Then if $\left\{\delta_{z}: z \in \mathbb{D}, \delta_{z} \in J^{\perp}\right\}$ is weak* dense in $\mathfrak{M}\left(H^{\infty}\right) \cap J^{\perp}$ (where $\mathfrak{M}\left(H^{\infty}\right.$ ) denotes the maximal ideal space of $\left.H^{\infty}\right)$, then the operators $q_{J} \circ C_{\tau}$ and $q_{J} \circ T$ agree on all of $H^{\infty}$. In this connection we remark that the famous Carleson Corona Theorem [14 guarantees the weak* density of $\left\{\delta_{z}: z \in \mathbb{D}\right\}$ in the maximal ideal space $\mathfrak{M}\left(H^{\infty}\right)$ of $H^{\infty}$, so that our condition seems interesting. Indeed, Carleson's theorem in this form gives another route to the characterization of unital type 1 isometries mentioned in the previous paragraph. This is work in progress, and details will be forthcoming.

Part II. Logmodularity and the $C^{*}$-envelope

\section{LOGMODULARITY AND REPRESENTING MEASURES}

A Dirichlet algebra is a unital subalgebra of $C(K)$ such that $A+\bar{A}$ is norm dense in $C(K)$, where here $\bar{A}$ denotes the set of adjoints of $A$. It follows that $A$ separates points of $K$, and thus, as is proved in most books on uniform algebras, $K=\partial A$, the Shilov boundary of $A$. We therefore define a (noncommutative) Dirichlet algebra to be a unital subalgebra $A$ of a unital $C^{*}$-algebra $B$, such that $\operatorname{Re}(A)$ is norm dense in $B_{s a}$. This is easily seen to be equivalent to $A+A^{*}$ being norm dense in $B$.

In order to define a noncommutative version of logmodularity, we will first recall a few basic facts about $C^{*}$-algebras. First, in a unital $C^{*}$-algebra, an element $a$ is strictly positive if and only if it is positive and invertible, and if and only if $b \geq \epsilon I$ for some real $\epsilon>0$. It will be helpful also to recall the following fact (which may be proved, for example, with the assistance of 41, 1.3.8): If $b$ is a strictly positive element in a unital $C^{*}$-algebra, and if $a_{n}$ is a sequence of positive elements in the algebra, then $a_{n} \rightarrow b$ uniformly (i.e., in the norm topology) if and only if $\sqrt{a_{n}} \rightarrow \sqrt{b}$.

We now proceed to define several related concepts, valid for a unital subalgebra $A$ of a unital $C^{*}$-algebra $B$. First, we say that $A$ has factorization, if each strictly positive $b \in B$ may be written as $a^{*} a$ for some $a \in A^{-1}$. This notion has been studied by many authors (e.g., 44, 46]). Next, we say that $A$ is left approximating in modulus (resp. left convexly approximating in modulus) if every positive $b \in B$ is a uniform limit of terms of the form $a^{*} a$ for an $a \in A$ (resp. $\sum_{k=1}^{n} a_{k}^{*} a_{k}$ for $a_{k} \in A$, $n$ varying too). Thus if $P=\left\{a^{*} a: a \in A\right\} \subset B_{+}$, then $A$ is left approximating in modulus (resp. left convexly approximating in modulus) if and only if $\bar{P}=B_{+}$ (resp. $\overline{\operatorname{co}\{P\}}=B_{+}$). The word 'left' here refers to the preference of products $a^{*} a$ as opposed to $a a^{*}$; thus right approximating in modulus means that each positive $b \in B$ is a uniform limit of terms of the form $a a^{*}$ for $a \in A$. If the word left or right is omitted, then we mean that both left and right hold.

We define a (noncommutative) logmodular algebra to be a unital subalgebra $A$ of a unital $C^{*}$-algebra $B$ such that every strictly positive element $b \in B$ is a uniform limit of terms of the form $a^{*} a$, where $a \in A^{-1}$.

Some well-known results for algebras with 'factorization' carry over to the 'logmodular case':

Proposition 4.1. Let $A$ be a unital subalgebra of a unital $C^{*}$-algebra $B$. The following are equivalent: 
(i) A has factorization, i.e., every strictly positive element $b \in B$ may be factored $b=a^{*} a$ for some $a \in A^{-1}$.

(ii) Every strictly positive element $b \in B$ equals $|a|$ for some $a \in A^{-1}$.

(iii) Every $b \in B^{-1}$ equals $u|a|$ for an $a \in A^{-1}$ and a unitary $u \in B$ (in fact, $u$ may be taken to be the unitary in the polar decomposition of $b$ ).

(iv) Every $b \in B^{-1}$ equals ua for an $a \in A^{-1}$ and a unitary $u \in B$.

Consequently, if $A$ has factorization, and if $b \in B^{-1}$, then $b A b^{-1}=u A u^{*}$ for $a$ unitary $u \in B$.

Also the following are equivalent:

(i)' $A$ is logmodular.

(ii)' Every strictly positive element $b \in B$ is a uniform limit of terms of the form $|a|$ for $a \in A^{-1}$.

(iii) ${ }^{\prime}$ Every $b \in B^{-1}$ is a uniform limit of terms of the form $u|a|$, where $a \in A^{-1}$ and $u$ is a unitary in $B$ (in fact, $u$ may be taken to be the unitary in the polar decomposition of $b$ ).

(iv)' Every $b \in B^{-1}$ is a uniform limit of terms of the form ua, where $a \in A^{-1}$ and $u$ is a unitary in $B$.

Proof. That (i) is equivalent to (ii) is obvious, whereas the fact that (i)' is equivalent to (ii) ${ }^{\prime}$ follows from the remarks made in the second paragraph of this section.

If $b \in B^{-1}$, then $b^{*} b \in B^{-1}$. Thus $b^{*} b$, and consequently $|b|$, is strictly positive. We may polar decompose $b=u|b|$, with $u$ a unitary in $B$. Supposing (i) to be true, we may write $b^{*} b=a^{*} a$ for $a \in A^{-1}$, giving $b=u|a|$, and thereby establishing (iii). Given (iii), if $a=w|a|$ is the polar decomposition of $a$, then $b=u w^{*} a$, giving (iv). Given (iv) and a strictly positive $b$, then $\sqrt{b}=u a$ for an $a \in A^{-1}$ and unitary $u$, so that $b=a^{*} a$ as in (i). The other assertions are similarly proved.

Parts (i)-(iv) are essentially in [5], 44] (see section 4.2 in [5] for example, where it is explained that the $u$ in (iv) is unique in a certain sense).

There seems to be a concept situated somewhere between logmodularity and convexly approximating in modulus (see [13]): we say that a uniformly closed unital subalgebra $A$ of a unital $C^{*}$-algebra $B$ is logrigged if every strictly positive element $b \in B$ is a uniform limit of terms of the form $\sum_{k=1}^{n} a_{k}^{*} a_{k}$, with $a_{k} \in A$, and $n$ varying too, where there exists $b_{k} \in A$ with $\sum_{k=1}^{n} b_{k} a_{k}=1$, and with the expressions $\sum_{k=1}^{n} b_{k} b_{k}^{*}$ converging uniformly to $b^{-1}$. By the remark in the second paragraph at the start of this section, this is equivalent to saying that every strictly positive $b \in B$ is a uniform limit of terms $\left(\sum_{k=1}^{n} a_{k}^{*} a_{k}\right)^{\frac{1}{2}}$, and $b^{-1}$ is a uniform limit of $\left(\sum_{k=1}^{n} b_{k} b_{k}^{*}\right)^{\frac{1}{2}}$, where $a_{k}, b_{k} \in A$ with $\sum_{k=1}^{n} b_{k} a_{k}=1$ ( $n$ varying too).

As yet we have not been able to construct an example of an algebra that is logrigged but not logmodular. Nonetheless, since several of our proofs below are not more difficult for logrigged algebras than for logmodular algebras, we will state these results for the former class.

There are various relationships between these notions, some of which are trivial (e.g., 'approximating in modulus' $\Rightarrow$ 'convexly approximating in modulus'). The main implications to bear in mind are:

Proposition 4.2. If $A$ is a unital subalgebra of a unital $C^{*}$-algebra, then we have the following implications:

'Factorization' $\Rightarrow$ 'logmodular' $\Rightarrow$ 'logrigged' $\Rightarrow$ 'convexly approximating in modulus'. 
Proof. The proofs of the first two implications are trivial. To see the last implication, one needs to note also that if $b \in B, b \geq 0$, then the terms $b+\frac{1}{n} 1$ are strictly positive and converge uniformly to $b$.

Remarks and examples. 1). For a function algebra $A$, the definitions above coincide with the classical ones. 'Factorization' is sometimes called 'strongly logmodular'. The term 'convexly approximating in modulus' was used in the function algebra case by Douglas and Paulsen.

2 ). The condition in the definition of a (noncommutative) logmodular algebra that every strictly positive $b$ is a uniform limit of terms of the form $a^{*} a$ implies that every strictly positive $b$ is a uniform limit of terms $a a^{*}$. This follows by replacing $b$ with $b^{-1}$, and noting that the inverse of $a a^{*}$, if $a$ is invertible in $A$, is $\left(a^{-1}\right)^{*} a^{-1}$. Thus the 'logmodular' condition is more symmetric than it appears at first sight, and there is no need to consider 'left' or 'right' logmodular.

A similar remark holds for the 'factorization' and 'logrigged' definitions.

3 ). The algebra of $n \times n$ upper triangular matrices is Dirichlet, and is known to have factorization (this is the Choleski factorization). Thus it is logmodular. This can be generalized to certain nest algebras (see e.g. 44], 46]). The $H^{\infty}$ algebras of [49] and their noncommutative generalization in [5] have factorization.

4). Logmodular and logrigged algebras usually furnish examples of the strong Morita equivalence of the first author with Muhly and Paulsen, as may be seen by the ideas in [13] (see e.g. the end of Section 6 there).

Proposition 4.3. Suppose that $A$ is a unital subalgebra of a unital $C^{*}$-algebra $B$, which either is Dirichlet, or is left or right convexly approximating in modulus. Then $B=C_{e}^{*}(A)$.

Proof. The 'Dirichlet' assertion is in [6], but in any case is immediate: namely, the canonical *-epimorphism $B \rightarrow C_{e}^{*}(A)$ is, by Lemma 1.2 an isometry and therefore is $1-1$.

Suppose that $A$ is left convexly approximating in modulus (the 'right' case will be similar). By the Arveson-Hamana theorem mentioned in the introduction, there is a *-homomorphism $\pi$ from $B$ onto $C_{e}^{*}(A)$. Let $I$ be the kernel of $\pi$; we will show that $I=\{0\}$. Let $q$ be the canonical map $q: A \rightarrow B / I$, factoring through the canonical maps $A \rightarrow B \stackrel{q_{I}}{\rightarrow} B / I$. Since the complete isometry $\pi_{\left.\right|_{A}}=j$ factors through $q$, it follows that $q$ is a complete isometry.

Suppose that $b \in I$ with $b \geq 0$. Then $b$ is a limit of terms of the form $\sum_{k=1}^{n} a_{k}^{*} a_{k}$, and hence $q_{I}(b)$ is the limit of terms $\sum_{k=1}^{n} q_{I}\left(a_{k}\right)^{*} q_{I}\left(a_{k}\right)=\sum_{k=1}^{n} q\left(a_{k}\right)^{*} q\left(a_{k}\right)$, since $q_{I}$ is a ${ }^{*}$-homomorphism. Also, $\|b\|$ is a limit of terms $\left\|\sum_{k=1}^{n} a_{k}^{*} a_{k}\right\|$. However, the last quantity is the square of the norm of the column in the column-space $C_{n}(A)$ (that is, the operator space given by the first column of $M_{n}(A)$ ) whose $k$ th entry is $a_{k}$. Since $q$ is a complete isometry, this norm coincides with the square of the norm of the column in $C_{n}(B / I)$ whose $k$ th entry is $q\left(a_{k}\right)$. Thus

$$
\|b\|=\lim \left\|\sum_{k=1}^{n} q\left(a_{k}\right)^{*} q\left(a_{k}\right)\right\|=\left\|q_{I}(b)\right\|=0 .
$$

Clearly $b=0$, which implies that $I=\{0\}$. 
Below we use the fact, alluded to in the introduction, that a unital linear map $S$ between operator systems (or unital $C^{*}$-algebras) is completely contractive if and only if it is completely positive, in which case it is *-linear, i.e., $S\left(v^{*}\right)=S(v)^{*}$.

Theorem 4.4. Suppose that $A$ is either a Dirichlet or a logrigged subalgebra of a unital $C^{*}$-algebra $B$. Then any unital completely contractive (resp. completely isometric) homomorphism $\varphi: A \rightarrow B(H)$ admits a unique extension to a completely positive and completely contractive (resp. completely isometric) map $B \rightarrow B(H)$. More generally, if $C$ is a unital operator algebra and if $\varphi: A \rightarrow C$ is a unital contractive homomorphism which possesses an extension to a completely positive map $B \rightarrow C$, then that extension is unique.

Proof. In the Dirichlet algebra case the claim regarding the existence of an extension follows immediately from Lemma 1.2 So assume that $A$ is logrigged. By the injectivity of $B(H)$, there does exist a completely positive extension to $B$.

To prove the claim regarding uniqueness, suppose that $\Phi$ and $\Psi$ are two completely positive extensions of $\varphi: A \rightarrow C$ to all of $B$. Since $C$ is a unital subalgebra of some $B(H)$, it suffices to consider only the case $C=B(H)$. Let $\xi$ be a unit vector in $H$, and suppose that $a_{k}, b_{k} \in A$ with $\sum_{k=1}^{n} b_{k} a_{k}=1$. Then

$$
1=\langle\xi, \xi\rangle=\left\langle\varphi\left(\sum_{k=1}^{n} b_{k} a_{k}\right) \xi, \xi\right\rangle=\sum_{k=1}^{n}\left\langle\Phi\left(b_{k}\right) \Psi\left(a_{k}\right) \xi, \xi\right\rangle .
$$

Using the Cauchy-Schwarz inequality in a standard way yields

$$
1 \leq\left\langle\sum_{k=1}^{n} \Psi\left(a_{k}\right)^{*} \Psi\left(a_{k}\right) \xi, \xi\right\rangle\left\langle\sum_{k=1}^{n} \Phi\left(b_{k}\right) \Phi\left(b_{k}\right)^{*} \xi, \xi\right\rangle,
$$

which by the Kadison-Schwarz inequality (see [19], 5.2.2) gives

$$
1 \leq\left\langle\Psi\left(\sum_{k=1}^{n} a_{k}^{*} a_{k}\right) \xi, \xi\right\rangle\left\langle\Phi\left(\sum_{k=1}^{n} b_{k} b_{k}^{*}\right) \xi, \xi\right\rangle .
$$

Since $A$ is logrigged, this yields

$$
1 \leq\langle\Psi(b) \xi, \xi\rangle\left\langle\Phi\left(b^{-1}\right) \xi, \xi\right\rangle
$$

for all strictly positive $b \in B$. Writing $b=e^{u}$ for $u \in B_{\text {sa }}$, we may then replace $u$ by $t u$ for real $t$, to obtain

$$
1 \leq\left\langle\Psi\left(e^{t u}\right) \xi, \xi\right\rangle\left\langle\Phi\left(e^{-t u}\right) \xi, \xi\right\rangle .
$$

Let $f(t)=\left\langle\Psi\left(e^{t u}\right) \xi, \xi\right\rangle\left\langle\Phi\left(e^{-t u}\right) \xi, \xi\right\rangle$, and differentiate as in the classical proof (see e.g. 17.1 in $[50])$. We get $f^{\prime}(0)=0$, which gives $\langle\Psi(u) \xi, \xi\rangle=\langle\Phi(u) \xi, \xi\rangle$. Hence $\Psi(u)=\Phi(u)$ for all selfadjoint $u \in B$. From this the claim regarding uniqueness of extensions is clear.

If $\varphi$ is completely isometric, then so is any extension of it to $B$ by the 'essential' property of $C_{e}^{*}(A)$ (see [22]).

Remark. There is a version of the above result for general unital contractions. In the Dirichlet case, essentially the same argument shows that such a map admits a unique positive extension to all of $B$. In proving the uniqueness of extension in the logrigged case, note that we only used the fact that $\Phi$ and $\Psi$ are continuous and satisfy the Kadison-Schwarz inequality $\Phi(x)^{*} \Phi(x) \leq \Phi\left(x^{*} x\right)$ for all $x \in A \cup A^{*}$. 
Corollary 4.5. Suppose that $A$ is a Dirichlet or logrigged subalgebra of a unital $C^{*}$-algebra $B$. Then (on relaxing the irreducibility requirement in [6]) every *representation of $B$ is a 'boundary representation' for $A$ in the language of [6].

Recall that in the commutative situation, the irreducible boundary representations of a uniform algebra $A \subset C(K)$ are precisely the point evaluations $\epsilon_{x}$ for $x \in K$ whose restrictions to $A$ possess a unique representing measure. Such points $x$ comprise precisely the Choquet boundary of $A$, and the closure of the Choquet boundary is the Shilov boundary. Since the 'representing measures' above are precisely completely positive maps extending the restriction of $\epsilon_{x}$ to $A$, it is clear that our last corollary may be interpreted as saying in some sense that for a Dirichlet or logrigged algebra, "the noncommutative Choquet boundary equals the Shilov boundary". In this connection we remark too that from the last corollary and facts in [6] one may deduce that $B$ has the universal property of $C_{e}^{*}(A)$, without having to use Hamana's theory.

Suppose that $A$ is a unital subalgebra of a unital $C^{*}$-algebra $B$. Then if $x \in$ $B^{-1}$, the algebra $x A x^{-1}$ is also a unital subalgebra of $B$, called a similarity of $A$. Similarity obviously does not arise in the commutative case, but it is very natural in the noncommutative case.

One question which seems interesting is the following: if $B$ is the $C^{*}$-envelope of $A$ (that is, if $\{0\}$ is the 'Shilov boundary ideal' in $B$ for $A$ ), and if $x$ is invertible in $B$, then what is the $C^{*}$-envelope of $x A x^{-1}$ ? Note that the $C^{*}$-subalgebra of $B$ generated by $x A x^{-1}$ need not be $B$. (For an example of this, let $A$ be the subalgebra of $M_{2}$ generated by $I_{2}$ and the matrix with first row having equal entries and second row composed of zeroes. This is similar to the diagonal two-dimensional $C^{*}$-algebra.) However, in a special case there is a nice answer:

Proposition 4.6. If $A$ is a unital subalgebra of a unital $C^{*}$-algebra $B$, and if $x \in B^{-1}$, then $x A x^{-1}$ has factorization (resp. is logmodular) if and only if $A$ has factorization (resp. is logmodular). If $A$ has factorization or is logmodular, then $C_{e}^{*}\left(x A x^{-1}\right)=B$ for all $x \in B^{-1}$.

Proof. Suppose that $A$ has factorization, and $x \in B^{-1}$. By Proposition 4.1 $x A x^{-1}=u A u^{*}$ for a unitary $u \in B$. If $b$ is a strictly positive element of $B$, then $u^{*} b u$ is strictly positive, and so equals $a^{*} a$ for $a \in A^{-1}$. Thus $b=u a^{*} a u^{*}=$ $\left(u a u^{*}\right)^{*}\left(u a u^{*}\right)$, so that $x A x^{-1}=u A u^{*}$ has factorization.

Next suppose that $A$ is $\operatorname{logmodular}$. In this case we know that $x=\lim _{n} u_{n} a_{n}$, where $u_{n}$ is unitary in $B$ and $a_{n} \in A^{-1}$; and it follows that $\lim _{n} a_{n}^{-1} u_{n}^{*}=x^{-1}$, and that $\left\{\left\|a_{n}^{-1}\right\|\right\}$ is bounded above. If $b$ is strictly positive in $B$, then for any fixed $n \in \mathbb{N}$ we have $u_{n}^{*} b u_{n}=\lim _{m}\left(a_{m}^{n}\right)^{*} a_{m}^{n}$, for some $\left\{a_{m}^{n}\right\}_{m} \subset A^{-1}$, so that $b=\lim _{m}\left(u_{n} a_{m}^{n} u_{n}^{*}\right)^{*}\left(u_{n} a_{m}^{n} u_{n}^{*}\right)$. Since $\left\|u_{n}^{*} b u_{n}\right\|=\|b\|$ for every $n$, we may select the $a_{m}^{n}$ 's so that the entire collection $\left\{a_{m}^{n}\right\}_{n, m}$ is uniformly bounded. Thus if $c_{m}^{n}=$ $a_{n}^{-1} a_{m}^{n} a_{n} \in A^{-1}$, then the collection $\left\{c_{m}^{n}\right\}_{n, m}$ is uniformly bounded, and it follows that there exist constants $K_{1}$ and $K_{2}$ such that

$$
\left\|u_{n} a_{n} c_{m}^{n} a_{n}^{-1} u_{n}^{*}-x c_{m}^{n} x^{-1}\right\| \leq K_{1}\left\|u_{n} a_{n}-x\right\|+K_{2}\left\|a_{n}^{-1} u_{n}^{*}-x^{-1}\right\|,
$$

and so, by a triangle inequality argument,

$$
b=\lim _{n} \lim _{m}\left(u_{n} a_{n} c_{m}^{n} a_{n}^{-1} u_{n}^{*}\right)^{*}\left(u_{n} a_{n} c_{m}^{n} a_{n}^{-1} u_{n}^{*}\right)=\lim _{n} \lim _{m}\left(x c_{m}^{n} x^{-1}\right)^{*}\left(x c_{m}^{n} x^{-1}\right),
$$

where $c_{m}^{n} \in A^{-1}$. Thus $x A x^{-1}$ is logmodular. 
The assertion about the $C^{*}$-envelope follows immediately from Proposition 4.3

\section{Conditional expectations and noncommutative $H^{\infty}$ SPaCes}

We begin this section with a result on conditional expectations which we think is new. It is a well-known result due to Tomiyama (see [54]) that a unital contractive projection from a unital $C^{*}$-algebra $A$ onto a unital subalgebra $B$ is completely contractive, and moreover is a 'conditional expectation' in the sense that

$$
P\left(b_{1} a b_{2}\right)=b_{1} P(a) b_{2}
$$

for all $a \in A, b_{1}, b_{2} \in B$.

Proposition 5.1. A completely contractive unital projection of a unital operator algebra $A$ onto a unital subalgebra $B$ is a 'conditional expectation' in the sense that

$$
P\left(b_{1} a b_{2}\right)=b_{1} P(a) b_{2}
$$

for all $a \in A, b_{1}, b_{2} \in B$.

Proof. Let $P: A \rightarrow B$ be the projection. Let $i: B \rightarrow A$ be the inclusion. We will use the basic properties of the injective envelope, as may be found in [22], [19] say. Let $(I(A), J)$ and $(I(B), j)$ be the injective envelopes of $A$ and $B$ respectively, which may be chosen to be unital $C^{*}$-algebras, with $J, j$ unital completely isometric homomorphisms. We may extend $j \circ P \circ J^{-1}$ to a completely contractive unital map $\tilde{P}: I(A) \rightarrow I(B)$ with $\tilde{P} \circ J=j \circ P$. We may also extend $J \circ i \circ j^{-1}$ to a completely contractive unital map $\tilde{i}: I(B) \rightarrow I(A)$, with $\tilde{i} \circ j=J \circ i$. Thus $\tilde{P} \circ \tilde{i}=I d$ on $j(B)$, and hence, by the rigidity property of the injective envelope, $\tilde{P} \circ \tilde{i}=I d$ on $I(B)$. Thus $Q=\tilde{i} \circ \tilde{P}$ is a unital completely contractive projection from $I(A)$ onto a subspace of $I(A)$ which is completely order isomorphic to the $C^{*}$-algebra $I(B)$. We have for $b \in B, a \in A$ that

$$
Q(J(a))=\tilde{i}(\tilde{P}(J(a)))=\tilde{i}(j(P(a))=J(P(a)),
$$

and thus

$$
J(P(b a))=Q(J(b a))=Q(J(b) J(a))=Q(J(b) Q(J(a))),
$$

where the last step uses a well-known lemma of Choi and Effros (see the proof of 6.1.2 in [19]). It follows from the last displayed equations (used three times) that

$$
J(P(b a))=Q(J(b P(a)))=J(P(b P(a)))=J(b P(a)),
$$

from which the result is clear.

Remark. The above proof provides an extension of $P$ to a completely positive surjective map $\tilde{P}: I(A) \rightarrow I(B)$. One can say a little more. We use the notation of the proof above. First, if $C^{*}(J(B))$ is the $C^{*}$-algebra generated by $J(B)$ inside $C_{e}^{*}(A) \subset I(A)$, then $\tilde{P}$ is a ${ }^{*}$-homomorphism from $C^{*}(J(B))$ onto $C_{e}^{*}(B)$. To see this, note that $\tilde{i}$ is a complete order isomorphism from $I(B)$ onto Ran $Q$. Hence $\tilde{i}$ is a ${ }^{*}$-isomorphism. That is, $\tilde{i}\left(j\left(b_{1}\right) j\left(b_{2}\right)^{*} \cdots j\left(b_{n}\right)\right)=Q\left(J\left(b_{1}\right) J\left(b_{2}\right)^{*} \cdots J\left(b_{n}\right)\right)$, or in other words,

$$
\tilde{P}\left(J\left(b_{1}\right) J\left(b_{2}\right)^{*} \cdots J\left(b_{n}\right)\right)=j\left(b_{1}\right) j\left(b_{2}\right)^{*} \cdots j\left(b_{n}\right)
$$

for $b_{1}, \cdots, b_{n} \in B$. Thus indeed $\tilde{P}$ is a *-homomorphism $C^{*}(J(B)) \rightarrow C_{e}^{*}(B)$, and $\tilde{P}\left(C^{*}(J(B))\right)=C_{e}^{*}(B)$. Therefore also $Q\left(C^{*}(J(B))\right)=\tilde{i}\left(C_{e}^{*}(B)\right)$. It follows from 
a well-known lemma of Choi that $\tilde{P}\left(a_{1} x a_{2}\right)=\tilde{P}\left(a_{1}\right) \tilde{P}(x) \tilde{P}\left(a_{2}\right)$, for $x \in I(A)$ and $a_{1}, a_{2} \in C^{*}(J(B))$.

We also remark that almost all of the above is true with the same proof, even if $B=P(A)$ is not a subalgebra of $A$. The only change is that we must amend the displayed equation in the proposition to read

$$
P\left(a_{1} P\left(a_{2}\right)\right)=P\left(P\left(a_{1}\right) a_{2}\right)=P\left(P\left(a_{1}\right) P\left(a_{2}\right)\right)
$$

for all $a_{1}, a_{2} \in A$.

The following result was originally stated by Le Merdy [31] (see also 3.4 and 3.5 in [32]) with the additional hypothesis of 'separate weak* continuity' of the product. This hypothesis was removed by the first author in [11. However, in fact, Proposition 5.1 gives a much simpler way to remove the hypothesis.

Corollary 5.2 (Le Merdy and Blecher). If $A$ is a unital operator algebra with an operator space predual, then $A$ is completely isometrically isomorphic, via a homomorphism which is also a homeomorphism for the weak ${ }^{*}$ topologies, to a $\sigma$ weakly closed unital subalgebra of $B(H)$, for some Hilbert space $H$.

Proof. We mimic Tomiyama's slick proof of Sakai's theorem [54]. The dual of the canonical map $i: A_{*} \rightarrow A^{*}$ dualizes to a weak* continuous completely contractive unital projection $P: A^{* *} \rightarrow A$. Let $J=\operatorname{Ker} P$, a weak ${ }^{*}$ closed subspace of $A^{* *}$. Then $P$ is a conditional expectation (by the proposition), so that $J$ is an $\hat{A}-\hat{A}$ subbimodule of $A^{* *}$. Since the product on $A^{* *}$ is separately weak* continuous, $J$ is a 2-sided ideal of $A^{* *}$. Also, if $F, G \in A^{* *}$, then $F-P(F) \in J$, so that $F G-P(F) G \in$ $J$. Thus $P(F G)=P(P(F) G)=P(F) P(G)$. Thus $P$ is a homomorphism. By elementary Banach space duality principles we obtain a completely isometric unital surjective weak* continuous homomorphism from $A^{* *} / J$ onto $A$. By the KreinSmulian theorem, this homomorphism is a homeomorphism for the weak* topologies (which implies that the multiplication on $A$ is separately weak* continuous). The result now follows from a part of Le Merdy's earlier result (one which was also independently proved in [4]).

In passing, we remark that if one could show that Proposition 5.1 holds with the word 'completely' deleted, then the proof of Corollary 5.2 would give a purely Banach algebraic characterization of $\sigma$-weakly closed operator algebras.

We now discuss a situation related to logmodularity/factorization in which conditional expectations naturally arise. Let $A$ be a unital subalgebra of a unital $C^{*}$-algebra $B$. Using classical $H^{\infty}(\mathbb{D})$ as a model, we may take $\Delta(A)=A \cap A^{*}$ to be a noncommutative analogue of the complex scalar field. A comparison of this context with the commutative setting (see for example [21, chapter IV) suggests that at least one possible approach to a noncommutative theory of Hardy spaces would be in terms of some fixed homomorphism $\varphi: A \rightarrow \Delta(A)=A \cap A^{*}$ which is also a projection of $A$ onto $\Delta(A)$. An extension of such a homomorphism to a positive projection from all of $B$ onto $\Delta(A)$ may then be regarded as some sort of noncommutative representing measure of $\varphi$. The questions of existence and uniqueness of such "representing measures" and the possible role of logmodularity in ensuring these eventualities immediately present themselves.

Let $M$ be a von Neumann algebra possessing a faithful normal tracial state $\tau$ (which implies that $M$ is a 'finite von Neumann algebra'). We say that $\tau$ preserves a map $\Phi: S \subset M \rightarrow M$ if $\tau \circ \Phi=\tau$ on the domain $S$ of $\Phi$. 
Lemma 5.3. For a unital *-algebra $M$ with a faithful state $\tau$ and a unital *subalgebra $N$, there is at most one unital 'conditional expectation' from any unital $N$-invariant subset $S$ of $M$ containing $N$, onto $N$, which is preserved by $\tau$.

By ' $N$-invariant' we mean that $N S N \subset S$, and by 'conditional expectation' here we mean that $\Phi(a s b)=a \Phi(s) b$ for all $a, b \in N, s \in S$. Since $N$ is unital, the fact that $\Phi$ is such a conditional expectation can be shown to imply that $\Phi$ preserves the identity and also that $\Phi \circ \Phi=\Phi$.

Proof. Suppose that $\Psi$ is another 'conditional expectation' of $S$ onto $N$ that is preserved by $\tau$. Then, using the conditional expectation property, we have for $a \in S$ that

$$
\begin{aligned}
\tau(\mid \Phi( & \left.a)-\left.\Psi(a)\right|^{2}\right) \\
& =\tau\left(\Phi(a)^{*} \Phi(a)\right)-\tau\left(\Phi(a)^{*} \Psi(a)\right)-\tau\left(\Psi(a)^{*} \Phi(a)\right)+\tau\left(\Psi(a)^{*} \Psi(a)\right) \\
& =\tau\left(\Phi\left(\Phi(a)^{*} a\right)\right)-\tau\left(\Psi\left(\Phi(a)^{*} a\right)\right)-\tau\left(\Phi\left(\Psi(a)^{*} a\right)\right)+\tau\left(\Psi\left(\Psi(a)^{*} a\right)\right) \\
& =\tau\left(\Phi(a)^{*} a\right)-\tau\left(\Phi(a)^{*} a\right)-\tau\left(\Psi(a)^{*} a\right)+\tau\left(\Psi(a)^{*} a\right) \\
& =0 .
\end{aligned}
$$

Since $\tau$ is faithful, this shows that $\Psi=\Phi$ on $S$.

We turn now to Arveson's remarkable noncommutative generalization of the $H^{\infty}$ spaces. Let $M$ be a von Neumann algebra with a faithful normal tracial state $\tau$, and suppose that $A$ is a weak* closed unital subalgebra of $M$. Then $\Delta(A)=A \cap A^{*}$ is a von Neumann subalgebra, and it is known ([52]; V.2.36) that there exists a faithful normal conditional expectation $\Phi$ of $M$ onto $\Delta(A)$ that is preserved by $\tau$. We say that $A$ is a finite maximal subdiagonal algebrd, or a noncommutative $H^{\infty}$ space, if, further, $A+A^{*}$ is weak*-dense in $M$ and $\Phi$ is multiplicative on $A$. So in the philosophy of the above discussion, $\Phi$ is in effect a representing measure of its restriction to $A$.

Many examples of finite maximal subdiagonal algebras are given in [5], [34], 35], for example. See also the appropriate chapter in [43] for a recent survey of subdiagonal algebras. The weak* Dirichlet algebras of [49] may be shown to all be finite maximal subdiagonal algebras (one may show that in this case $\Delta(A)=\mathbb{C} 1$ ). If $A$ is a finite maximal subdiagonal algebra, then so is $M_{n}(A)$. In [5] it is shown that any finite maximal subdiagonal algebra has factorization. It is consequently logmodular, and we may apply the results proved in Section 4 above. From this and Proposition 4.3 we may deduce the following generalization of a well-known fact for classical $H^{\infty}(\mathbb{T})$ :

Proposition 5.4. If $A$ is a finite maximal subdiagonal algebra in $M$, then $M=$ $C_{e}^{*}(A)$. That is, $\{0\}$ is the noncommutative Shilov boundary ideal in $M$ of $A$.

Remark. It is well known that every commutative von Neumann algebra $M$ is an injective Banach space, and is hence an injective operator space. In this case we can say, under the hypotheses of the previous corollary, that $M$ is the injective envelope' of $A$ too. (This follows easily from abstract principles in [22] or [19.) This is interesting, because there are few cases where the injective envelope of an operator space is explicitly known.

\footnotetext{
${ }^{1}$ The simplified form of the definition is due to Exel.
} 
Definition 5.5. Let $M$ be a von Neumann algebra with a faithful normal tracial state $\tau$. A tracial subalgebra of $M$ is a weak* closed unital subalgebra $A$ of $M$ for which there exists a linear projection $\Psi$ from $A$ onto $\Delta(A)=A \cap A^{*}$ that is also a homomorphism on $A$, such that $\tau(a)=\tau(\Psi(a))$ for all $a \in A$.

Theorem 5.6. Let $A$ be a tracial subalgebra of a von Neumann algebra $M$. Then the map $\Psi$ in Definition 5.5 is unique, completely contractive, and weak* continuous. Indeed, $\Psi$ is the restriction to $A$ of the canonical conditional expectation of $M$ onto $\Delta(A)$ that is preserved by $\tau$. Furthermore,

(1) If $A+A^{*}$ is weak ${ }^{*}$ dense in $M$, then $A$ is a finite maximal subdiagonal algebra in $M$. Thus $A$ will then have factorization, and will be logmodular.

(2) If $A$ is logmodular or logrigged, then the canonical conditional expectation from $M$ onto $\Delta(A)$ is the only positive extension of $\Psi$ to a map from $M$ into $C$, for any $C^{*}$-algebra $C$ containing ( $a C^{*}$-algebra $*$-isomorphic to) $\Delta(A)$.

Proof. The conditional expectation from $M$ onto $\Delta(A)$ restricts to a 'conditional expectation' from $A$ onto $\Delta(A)$. Clearly $\Psi$ is a 'conditional expectation' from $A$ onto $\Delta(A)$. The first claim then follows by Lemma 5.3 as does (1).

To see (2), note that by the remark following Theorem 4.4 we only need to verify that each positive extension of $\Psi$ satisfies the Kadison-Schwarz inequality on $A \cup A^{*}$. To this end, let $C$ be a unital $C^{*}$-algebra, let $j: \Delta(A) \rightarrow C$ be a unital 1-1 *-homomorphism, and let $\Phi: M \rightarrow C$ be any positive extension of $j \circ \Psi$ to all of $M$. For simplicity the reader may want to take $C=M$ and $j=I d$ in the following. By taking adjoints we may conclude from $\Psi$ 's action on $A$ that $\Phi$ is unital, that it maps $A+A^{*}$ into $j(\Delta(A)$ ), satisfies the equality $\left.\Phi \circ j^{-1} \circ \Phi\right|_{A+A^{*}}=\left.\Phi\right|_{A+A^{*}}$, and also acts homomorphically on both $A$ and $A^{*}$. Therefore on expanding the term $\left(x^{*}-j^{-1} \Phi\left(x^{*}\right)\right)\left(x-j^{-1} \Phi(x)\right) \geq 0$ and applying $\Phi$, it follows that $\Phi\left(x^{*} x\right) \geq \Phi\left(x^{*}\right) \Phi(x)$ for each $x \in A \cup A^{*}$, as required.

We close our paper with an open problem: If $A$ is a logrigged or logmodular tracial subalgebra, when is $A^{*}+A$ automatically weak* dense in the ambient von Neumann algebra? We recall that in the setting of commutative weak* Dirichlet algebras considered by Srinivasan and Wang, it is known that logmodularity and the weak* density of $A^{*}+A$ in $L^{\infty}$ are equivalent.

We will give a result which probably is in the right direction towards the solution of this problem. In the commutative context of Srinivasan and Wang and other authors, one first proves $L^{2}$-density of $A^{*}+A$, before combining Szegö's theorem with this fact to conclude that in fact weak* density of $A^{*}+A$ in $L^{\infty}$ pertains. See also [33, where this is linked very tightly to the principle of Theorem 4.4. Thus our question seems tied to the open problems surrounding the apparent absence of a suitable version of 'Jensen's inequality' and 'Szegö's theorem' in the general noncommutative case (see [5], [35], and references contained therein). A weaker version of the aforementioned open problem may therefore be formulated as follows: Is weak* density of $A^{*}+A$ in the ambient von Neumann algebra equivalent to $\operatorname{logmodularity}$ of $A$ for all tracial algebras $A$ that satisfy a noncommutative Szegö theorem in the sense described in 4.4 of [5]?

Proposition 5.7. Let $A$ be a logrigged tracial subalgebra of a von Neumann algebra $M$, with $\Delta(A)$ contained in the center of $M$. Then $A+A^{*}$ is a dense subspace of $L^{1}(M, \tau)$. 
Proof. Suppose, by way of contradiction, that $A$ satisfies the hypotheses of the proposition, but that $A^{*}+A$ is not dense in $L^{1}(M, \tau)$. By the Hahn-Banach theorem and the duality of noncommutative $L^{p}$-spaces (see e.g. [53]), we would be able to find some $x \in M$ with $x \neq 0$ and $\tau(x a)=0$ for all $a \in A^{*}+A$. By taking adjoints it is easy to see that $\tau(x a)=0$ for all $a \in A^{*}+A$ if and only if $\tau\left(\left(x+x^{*}\right) a\right)=0$ and $\tau\left(\left(i\left(x^{*}-x\right)\right) a\right)=0$ for all $a \in A^{*}+A$. We may therefore assume $x$ to be a selfadjoint element of $M$, and, on suitably scaling $x$, even that $\|x\|_{\infty} \leq 1$. Then $1+x$ is a positive element of $M$.

If $d \in \Delta(A)$ and $a \in A^{*}+A$, then

$$
\tau(\Phi(x a) d)=\tau(\Phi(x a d))=\tau(x a d)=0 .
$$

Since this holds for all $d \in \Delta(A)$, we may conclude that $\Phi(x a)=0$ for $a \in A^{*}+A$. Consider the linear map $\Psi: a \mapsto \Phi((1+x) a)$ on $M$. This coincides with $\Phi$ on $A$. If $d \in \Delta(A)$ and $a \in M$, then, using the conditional expectation property, the fact that $\tau$ preserves $\Phi$, and the facts that $d$ is in the center and $\tau$ is a trace, we have

$$
\tau\left(d^{*} \Phi\left((1+x) a^{*} a\right) d\right)=\tau\left(\Phi\left(d^{*}(1+x) a^{*} a d\right)\right)=\tau\left(d^{*} a(1+x) a^{*} d\right) \geq 0 .
$$

Since this holds for all $d \in \Delta(A)$, it follows by elementary considerations that $\Phi\left((1+x) a^{*} a\right) \geq 0$. Thus $\Psi$ is positive, and therefore completely positive, seeing as its range is commutative [40. It follows from Theorem 4.4 that $\Phi((1+x) a)=\Phi(a)$ for all $a \in M$. Thus $\Phi(x M)=0$, which implies that $\Phi\left(x^{2}\right)=0$. Since $\Phi$ is faithful, this gives the contradiction $x=x^{2}=0$.

Acknowledgments. We thank Alec Matheson for drawing our attention to the area discussed in Part I above, and for helpful discussions and clarifications. We also thank the University of South Africa for supporting a visit of the first author.

Added in proof. For additional background and a survey of topics connected with Theorem 2.2, see [56], 57].

\section{REFERENCES}

[1] M. Anoussis and E. G. Katsoulis, Compact Operators and the Geometric Structure of Nest Algebras, Indiana. Univ. Math. J. 46 (1997), 319-336. MR 98e:47066b

[2] J. Araujo and J. J. Font, Linear isometries between subspaces of continuous functions, Trans. Amer. Math. Soc. 349 (1997), 413-428. MR 97d:46026

[3] J. Arazy and B. Solel, Isometries of nonselfadjoint operator algebras, J. Funct. Anal. 90 (1990), 284-305. MR 91c:47085

[4] A. Arias and G. Popescu, Noncommutative interpolation and Poisson transforms, Israel J. Math. 115 (2000), 205-235. MR 2001f:47021

[5] W. B. Arveson, Analyticity in operator algebras, Amer. J. Math. 89 (1967), 578-642. MR 36:6946

[6] W. B. Arveson, Subalgebras of $C^{*}$-algebras, Acta Math. 123 (1969), 141-22. MR 40:6274

[7] W. B. Arveson, Subalgebras of $C^{*}$-algebras II, Acta Math. 128 (1972), 271-308. MR 52:15035

[8] D. P. Blecher, Commutativity in operator algebras, Proc. Amer. Math. Soc. 109 (1990), 709-715. MR 90k:46128

[9] D. P. Blecher, Modules over operator algebras and the maximal $C^{*}$-dilation, J. Funct. Anal. 169 (1999), 251-288. MR 2000j: 47122

[10] D. P. Blecher, The Shilov boundary of an operator space, and the characterization theorems, J. Funct. Anal. 182 (2001), 280-343. MR 2002d:46049

[11] D. P. Blecher, Multipliers and dual operator algebras, J. Funct. Anal. 183 (2001), 498-525.

$[12]$ D. P. Blecher and D. Hay, Complete isometries into $C^{*}$-algebras, preprint (March '02), http://front.math.ucdavis.edu/math.OA/0203182 .

[13] D. P. Blecher and K. Jarosz, Isomorphisms of function modules, and generalized approximation in modulus, Trans. Amer. Math. Soc. 354 (2002), 3663-3701. 
[14] L. Carleson, Interpolation by bounded analytic functions and the corona problem, Ann. of Math. 76 (1962), 547-559. MR 25:5186

[15] M. D. Choi and E. G. Effros, The completely positive lifting problem for $C^{*}$-algebras, Ann. of Math. 104 (1976), 585-609. MR 54:5843

[16] C-H. Chu and N-C. Wong, Isometries between $C^{*}$-algebras, Rev. Mat. Iberoamericana, to appear.

[17] J. B. Conway, A Course in Operator Theory, Amer. Math. Soc., Providence, RI, 2000. MR 2001d:47001

[18] E. G. Effros and Z. J. Ruan, On non-self-adjoint operator algebras, Proc. Amer. Math. Soc. 110 (1990), 915-922. MR 91c:47086

[19] E. G. Effros and Z. J. Ruan, Operator Spaces, Oxford University Press, Oxford, 2000. MR 2002a: 46082

[20] M. El-Gebeily and J. Wolfe, Isometries of the disk algebra, Proc. Amer. Math. Soc. 93 (1985), 697-702. MR 86j:46058

[21] T. W. Gamelin, Uniform Algebras, $2^{\text {nd }}$ ed., Chelsea, New York, 1984. MR 53:14137 (1st ed.)

[22] M. Hamana, Injective envelopes of operator systems, Publ. Res. Inst. Math. Sci. 15 (1979), 773-785. MR 81h:46071

[23] M. Hamana, Triple envelopes and Silov boundaries of operator spaces, Math. J. Toyama Univ. 22 (1999), 77-93. MR 2001a:46057

[24] K. Hoffman, Analytic functions and logmodular Banach algebras, Acta Math. 108 (1962), 271-317. MR 26:6820

[25] K. Hoffman, Banach spaces of analytic functions, Dover, New York, 1988. MR 92d:46066

[26] K. Jarosz and V. Pathak, Isometries and small bound peturbations of function spaces. In: Function Spaces, Lecture Notes in Pure and Applied Math., Vol. 136, Marcel Dekker, New York, 1992, 241-271. MR 93b:47061

[27] R. V. Kadison, Isometries of operator algebras, Ann. of Math. 54 (1951), 325-338. MR 13:256a

[28] E. Kirchberg, On restricted peturbations in inverse images and a description of normalizer algebras in $C^{*}$-algebras, J. Funct. Anal. 129 (1995), 1-34. MR 95m:46094a

[29] L. E. Labuschagne, Analogues of composition operators on non-commutative $H^{p}$-spaces, J. Operator Theory, to appear.

[30] L. E. Labuschagne, Composition operators on non-commutative $L_{p}$-spaces, Expo. Math. 17 (1999), 429-468. MR 2001f:46099

[31] C. Le Merdy, An operator space characterization of dual operator algebras, Amer. J. Math. 121 (1999), 55-63. MR 2001f:46086

[32] C. Le Merdy, Finite rank approximations and semidiscreteness for linear operators, Ann. Inst. Fourier (Grenoble) 49 (1999), 1869-1901. MR 2001b:46092

[33] G. Lumer, Analytic functions and Dirichlet problems, Bull. Amer. Math. Soc. 70 (1964), 98-104. MR 28:1509

[34] M. McAsey, P. Muhly, and K.-S. Saito, Nonselfadjoint crossed products (invariant subspaces and maximality), Trans. Amer. Math. Soc. 248 (1979), 381-409. MR 80j:46101b

[35] M. Marsalli and G. West, Noncommutative $H^{p}$-spaces, J. Operator Theory 40 (1998), 339355. MR 2001b:46117

[36] A. Matheson, Isometries into function algebras, Houston J. Math., to appear.

[37] M. Nagasawa, Isomorphisms between commutative Banach algebras with an application to rings of analytic functions, Kodai Math. Sem. Rep. 11 (1959), 182-188. MR 22:12379

[38] W. P. Novinger, Linear isometries of subspaces of spaces of continuous functions, Studia Math. 53 (1975), 273-276. MR 54:5818

[39] D. P. O' Donovan and K. R. Davidson, Isometric images of $C^{*}$-algebras, Canad. Math. Bull. 27 (1984), 286-294. MR 85h:46081

[40] V. I. Paulsen, Completely bounded maps and dilations, Pitman Research Notes in Math., vol. 146, Longman, London, 1986. MR 88h:46111

[41] G. Pedersen, $C^{*}$-algebras and their automorphism groups, Academic Press, London, 1979. MR 81e:46037

[42] G. Pisier, Introduction to operator space theory, Cambridge University Press, to appear. (French original, MR 98e:46019) 
[43] G. Pisier and Q. Xu, Noncommutative $L^{p}$ spaces, Handbook on the geometry of Banach spaces (Vol. 2), to appear. Ed.: W. B. Johnson and J. Lindenstrauss.

[44] D. Pitts, Factorization problems for nests: factorization methods and characterizations of the universal factorization property, J. Funct. Anal. 79 (1988), 57-90. MR 90a:47101

[45] Y-t. Poon and Z. J. Ruan, Operator algebras with contractive approximate identities, Canad. J. Math. 46 (1994), 397-414. MR 95d:47057

[46] S. C. Power, Factorization in analytic operator algebras, J. Funct. Anal. 67 (1986), 413-432. MR 87k:47040

[47] D. Sarason, Composition operators as integral operators. In: Analysis and partial differential equations, Marcel Dekker, New York, 1990, 545-565. MR 92a:47040

[48] R. K. Singh and J. S. Manhas, Composition operators on function spaces, North-Holland, Amsterdam, 1993. MR 95d:47036

[49] T. P. Srinivasan and J-K. Wang, Weak*-Dirichlet algebras. In: Ed. Frank T. Birtel, Function algebras, Scott Foresman and Co., 1966, 216-249. MR 33:6441

[50] E. L. Stout, The theory of uniform algebras, Bogden and Quigley, Tarrytown-on-Hudson, NY, 1971. MR 54:11066

[51] I. Suciu, Function algebras, Noordhoff Internat. Pub., Leyden, 1975. MR 51:6428

[52] M. Takesaki, Theory of Operator Algebras I, Springer, New York, 1979. MR 81e:46038

[53] M. Terp, $L^{p}$-spaces associated with von Neumann algebras, Copenhagen University, 1981.

[54] J. Tomiyama, Tensor products and pojections of norm one in von Neumann algebras, lecture notes, Copenhagen University, 1970.

[55] C. Zhang, Representations of operator spaces, J. Operator Theory 33 (1995), 327-351. MR 96h:46092

[56] D. P. Blecher and D. M. Hay, Complete isometries - an illustration of noncommutative functional analysis, Provisionally accepted, Proceedings of the Fourth Conference on Function Spaces, Contemp. Math., Amer. Math. Soc., Providence, RI.

[57] R. J. Fleming and J. E. Jamison, Isometries on Banach spaces: function spaces, CRC Press, to appear.

Department of Mathematics, University of Houston, Houston, Texas 77204-3008

E-mail address: dblecher@math.uh.edu

Department of Mathematics, Applied Mathematics and Astronomy, P.O. Box 392, 0003 UNISA, South Africa

E-mail address: labusle@unisa.ac.za 